\title{
Polarized Single-Particle Quantum Dot Emitters through Programmable Cluster Assembly
}

Honghu Zhang ${ }^{1}$, Mingxing $\mathrm{Li}^{1}$, Kaiwei Wang ${ }^{1,2}$, Ye Tian ${ }^{1}$, Jia-Shiang Chen ${ }^{1}$, Katherine T. Fountaine ${ }^{3}$, Donald DiMarzio ${ }^{3}$, Mingzhao Liu ${ }^{1}$, Mircea Cotlet ${ }^{1}$, Oleg Gang ${ }^{* 1,4,5}$

1. Center for Functional Nanomaterials, Brookhaven National Laboratory, Upton, NY 11973, USA

2. School of Science, Xi'an Jiaotong University, Xi'an 710049, China

3. NG Next, Northrop Grumman Corporation, One Space Park, Redondo Beach, CA 90278, USA

4. Department of Chemical Engineering, Columbia University, New York, NY 10027, USA

5. Department of Applied Physics and Applied Mathematics, Columbia University, New York, NY 10027, USA

*Corresponding Author; e-mail: og2226@columbia.edu

\section{Supporting Information}

\section{Table of content}

1. Supplementary figures

2. Experimental methods

3. Structural characterizations

4. Optical characterizations

5. Theoretical considerations

6. DNA sequences 
1. Supplementary figures

a
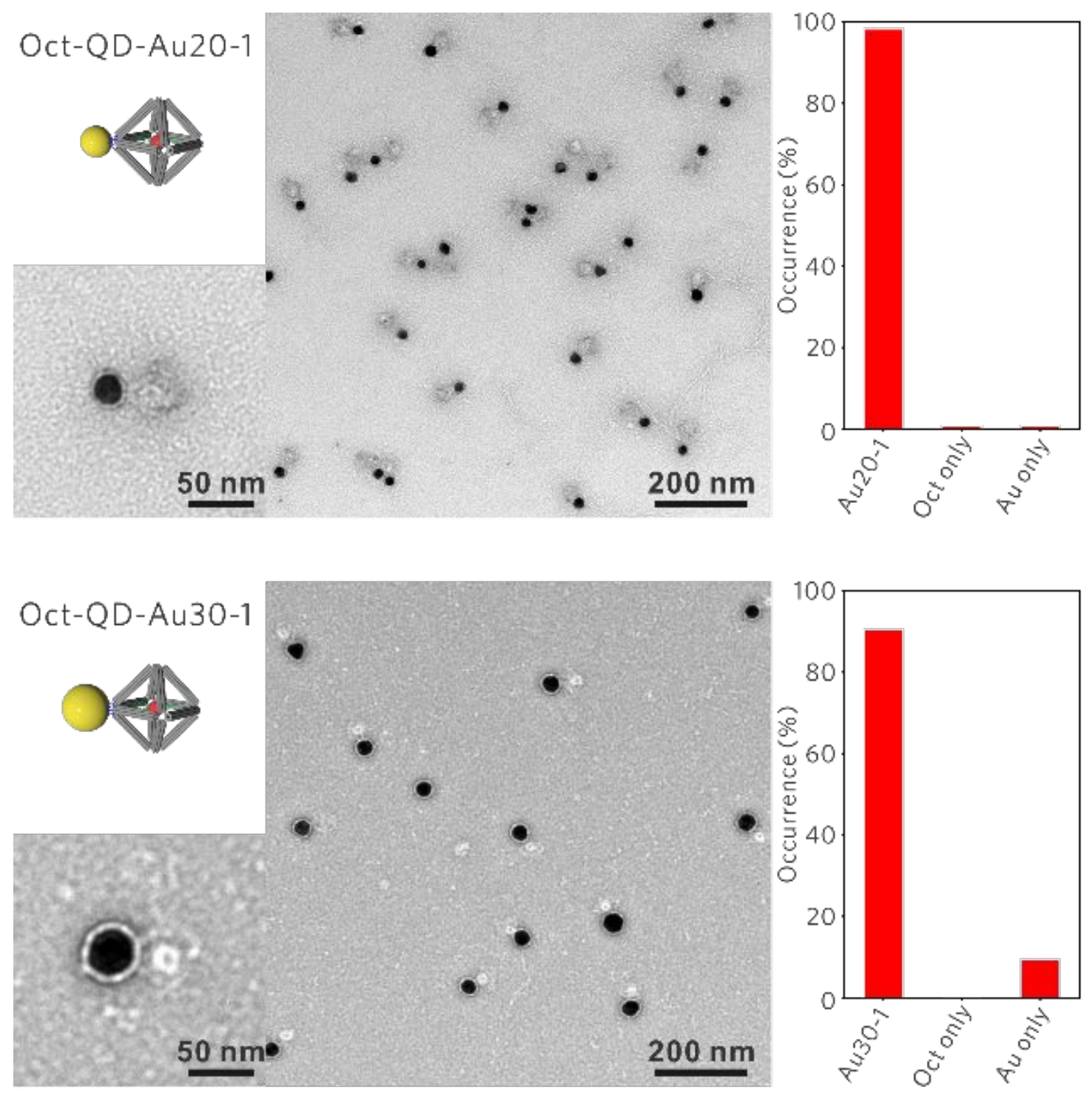
b
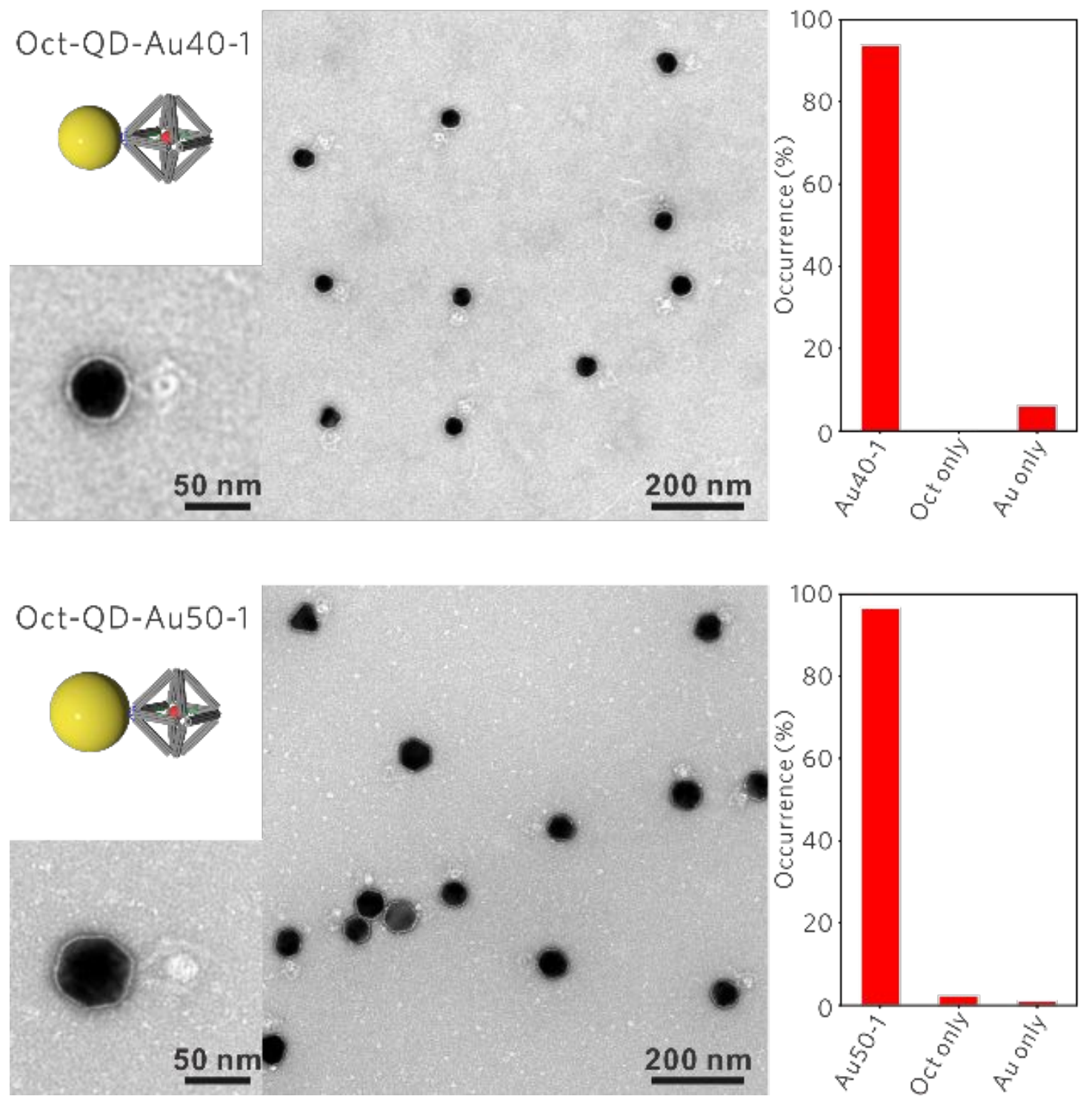
C
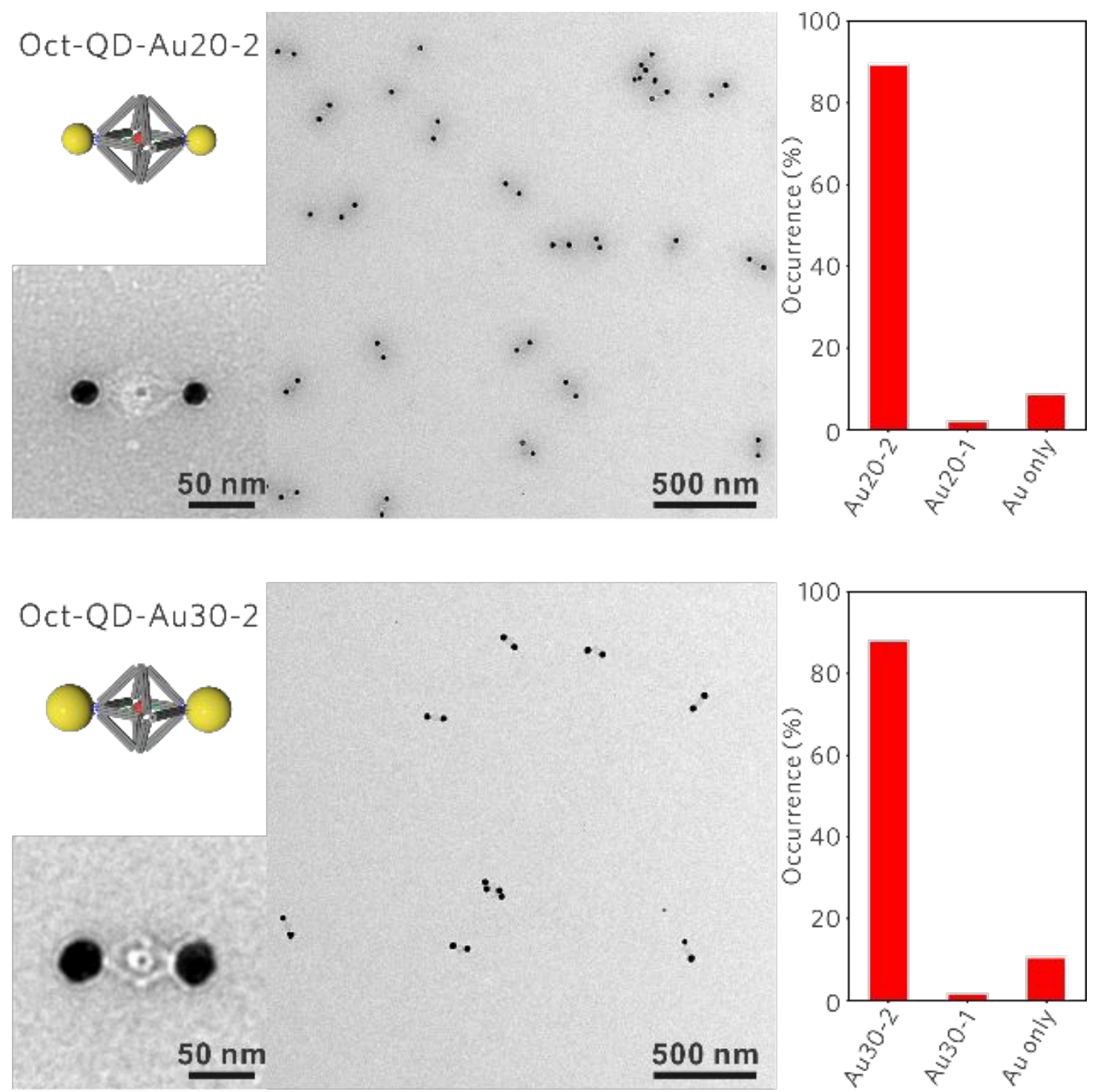

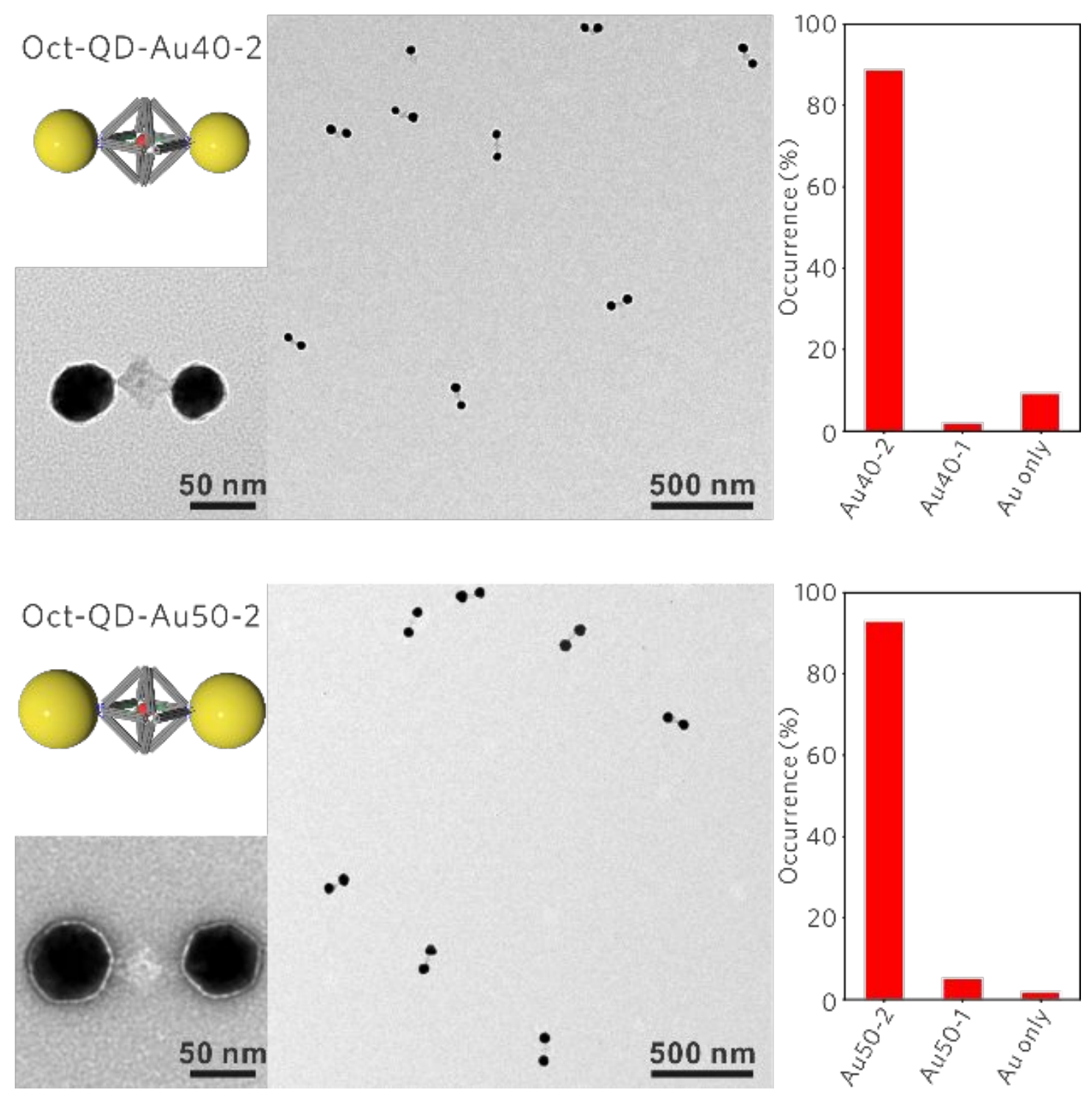

Figure S1. TEM images of the nanoclusters consisting of a single QD and a AuNP monomer (a-b) or a AuNP dimer (c-d) with the AuNP size range from 20 to $50 \mathrm{~nm}$ and the corresponding population histograms of the Oct-AuNP monomer or the Oct-AuNP dimer. Note: For measuring the occurrence of each structures, the presence of QDs is neglected due to much lower contrast of QDs than that of AuNPs; the final structural purity is expressed as the product of Oct-AuNP structural purity and the QD capsuling efficiency ( 82\% Fig. 1c). For each type of nanoclusters, 200-700 individual nanoclusters were surveyed in the TEM images. 

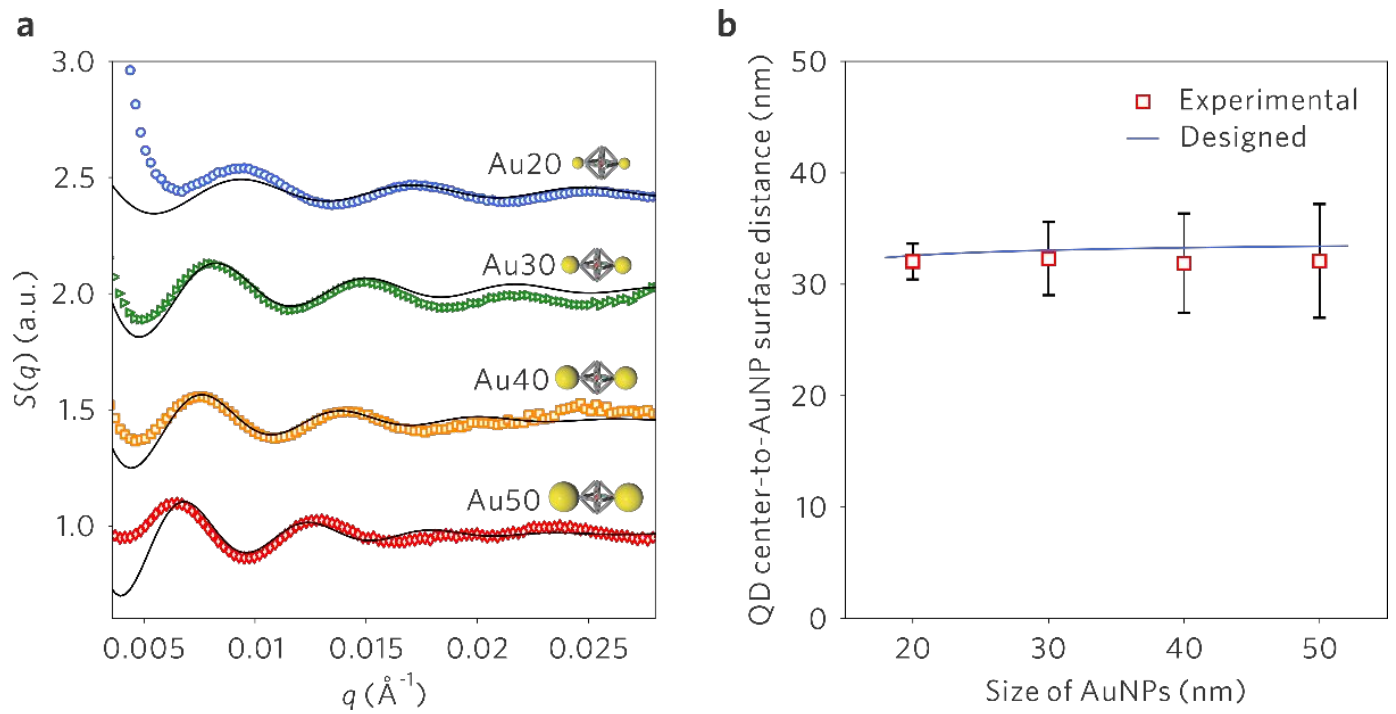

Figure S2. Characterization of interparticle distances of divalent nanoclusters in liquid suspension by small-angle X-ray scattering (SAXS). a, Extracted structure factor $S(q)$ for the divalent nanoclusters with various AuNP sizes. Black solid lines are best fits with a dumbbell model which is described below in Structural characterization section. The plots are vertically shifted for display purpose. b, The characteristic distance, i.e., QD center-to-AuNP surface distance, obtained from the SAXS measurements and the nanocluster design (see details below). The QD center-to-AuNP surface distance is approximately $32 \mathrm{~nm}$. The error bars show the distance spreads, where mainly originate from size polydispersity of AuNPs. 


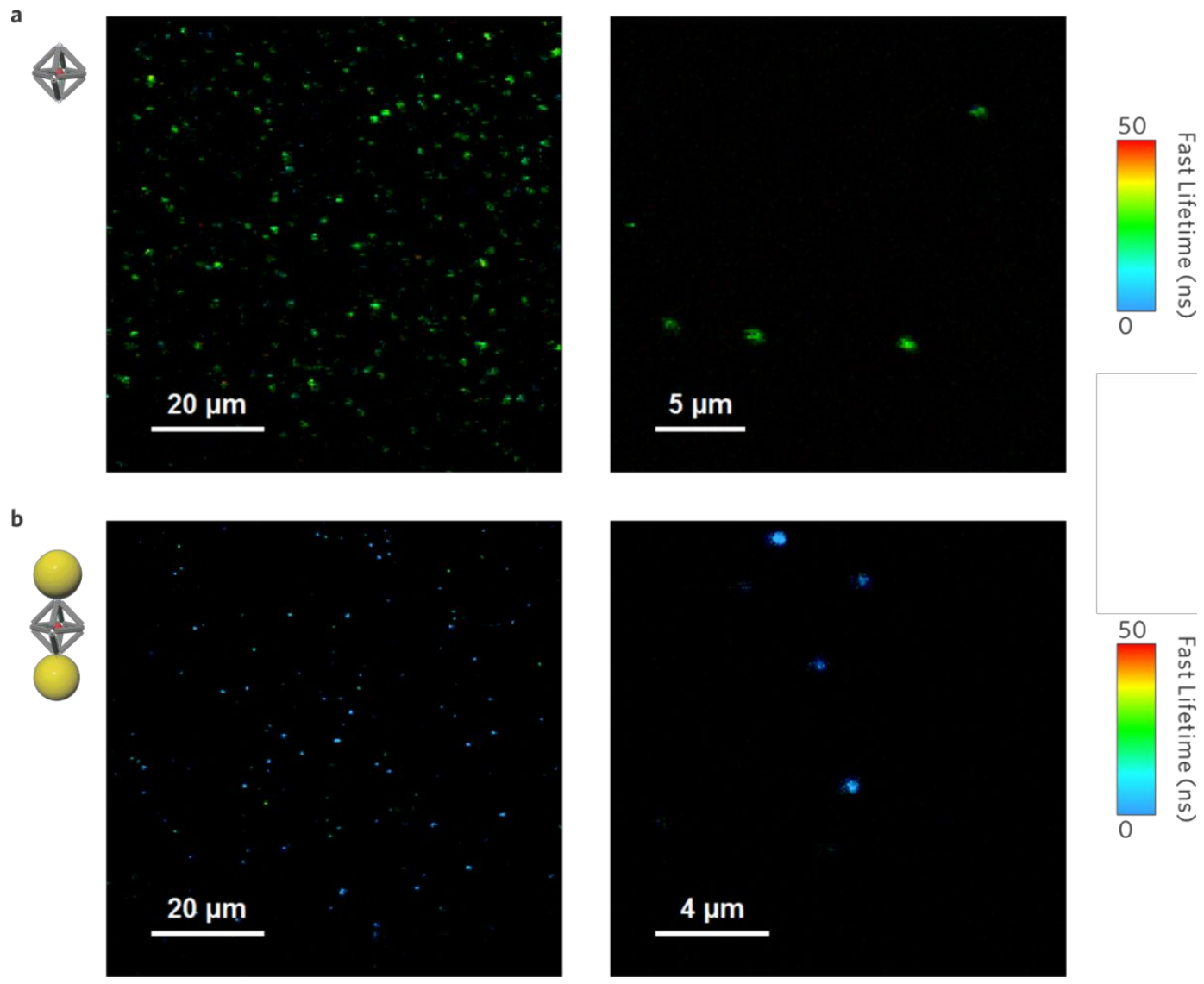

Figure S3. Typical FLIM images on large and small scan areas of the Oct-QD (a) and the Oct-QD-Au50-2 nanoclusters (b). The FLIM images show that the Oct-QD-Au50-2 nanoclusters exhibit much shorter lifetime than the Oct-QD. 
a

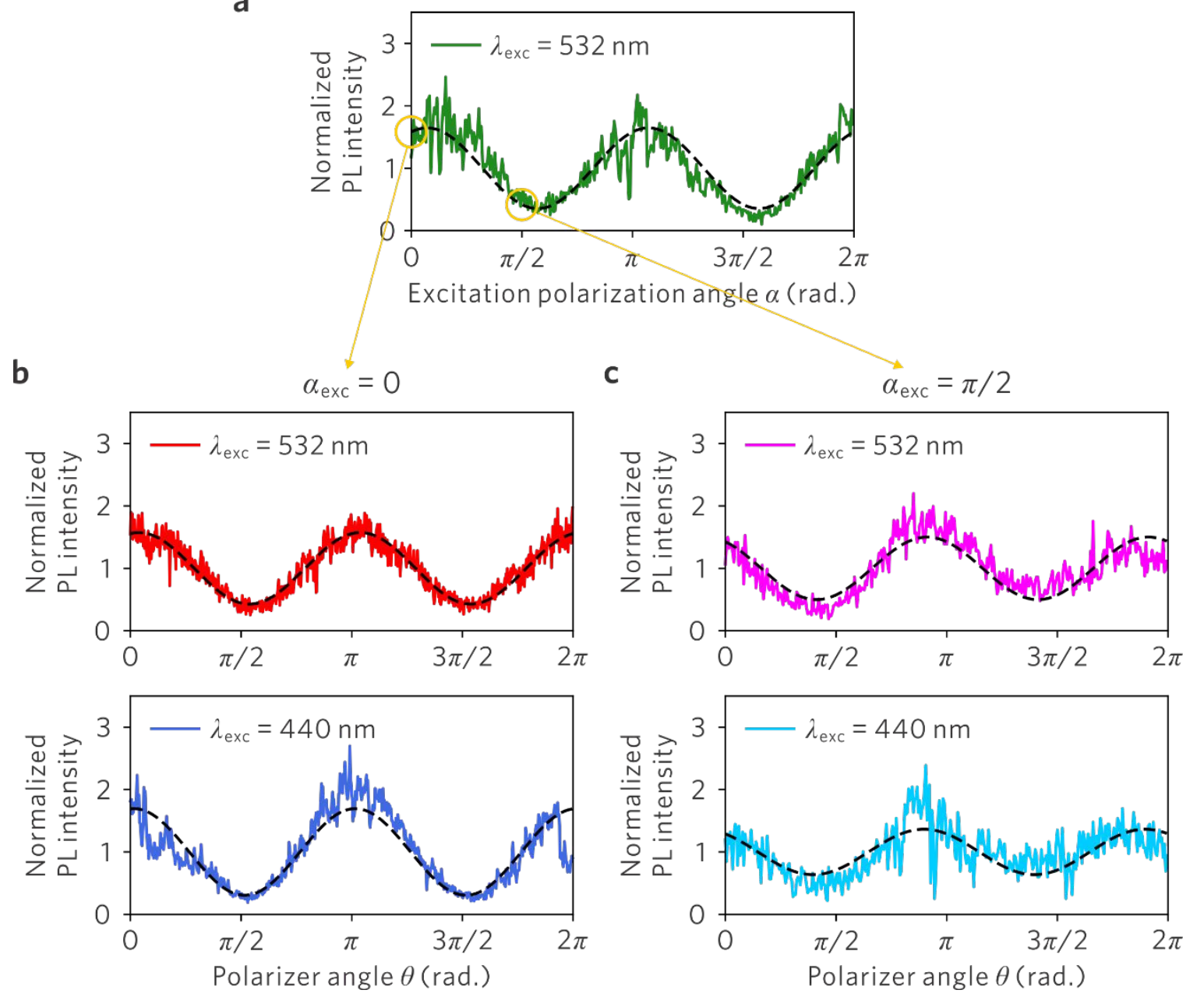

Figure S4. The excitation polarization and emission polarization measurements for an individual Oct-QDAu50-2 nanocluster in solution. a, The PL intensity profile as a function of excitation polarization angles $\left(\alpha_{\text {exc }}\right)$ at $\lambda_{\text {exc }}=532 \mathrm{~nm}$. b. At fixed excitation polarization angle, $\alpha_{\text {exc }}=0$, the PL intensity profiles as function of polarizer angles $(\theta)$ for $\lambda_{\text {exc }}=532 \mathrm{~nm}$ and $440 \mathrm{~nm}$. c, At fixed excitation polarization angle, $\alpha_{\text {exc }}=\pi / 2$, the PL intensity profiles as function of polarizer angles $\theta$ for $\lambda_{\text {exc }}=532 \mathrm{~nm}$ and $440 \mathrm{~nm}$. 

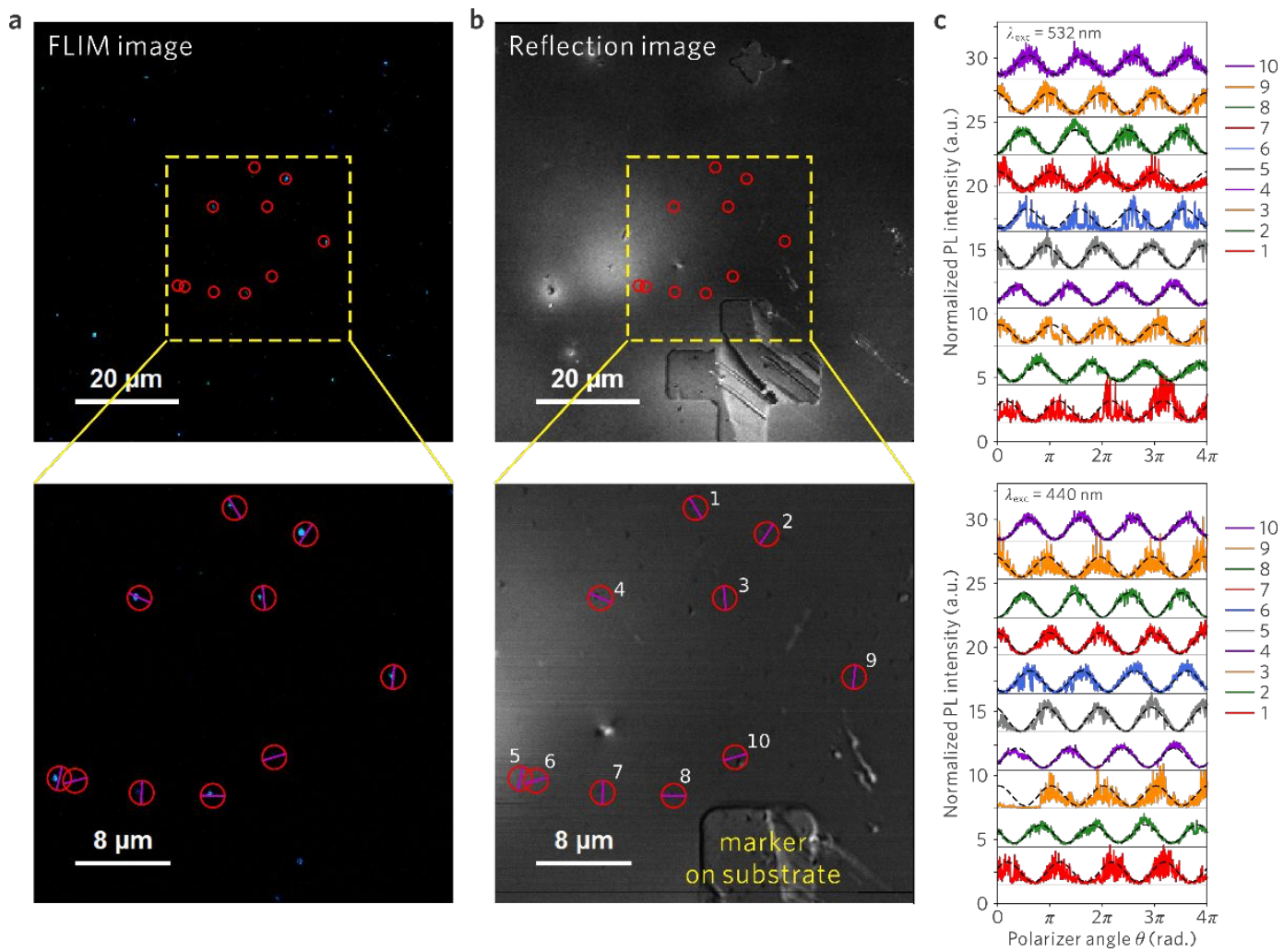

Figure S5. Emission polarization detections of Oct-QD-Au50-2 nanoclusters deposited and dried on the silicon substrate. a, Confocal FLIM images on large and small scan areas. b, Corresponding reflection images on large and small scan areas. The measured nanoclusters are circled in each image and labeled with their position number (No. 1-10). c, PL intensity profiles as a function of emission polarization angles at two excitation wavelengths $\left(\lambda_{\text {exc }}=532 \mathrm{~nm}\right.$ and $\left.440 \mathrm{~nm}\right)$. The plots are shifted vertically for clarify. The polarization directions determined from the phases are shown in the small-scan confocal FLIM image and reflection image. 
a

FLIM image

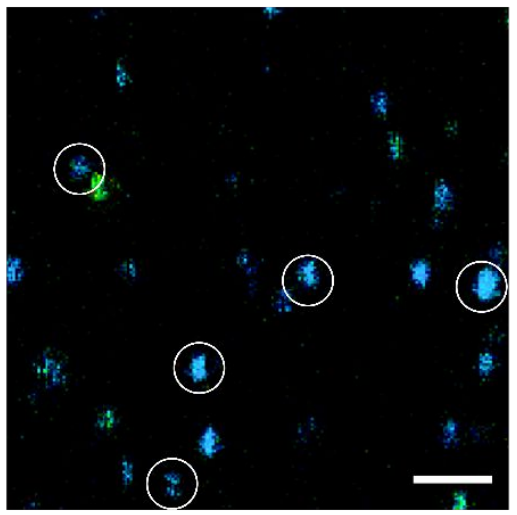

c Emission polarization

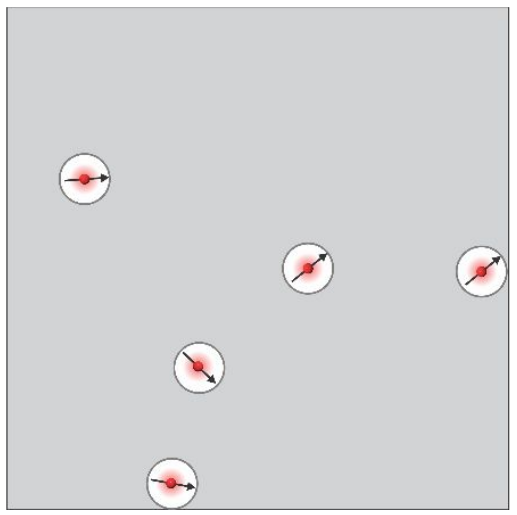

b

Reflection image

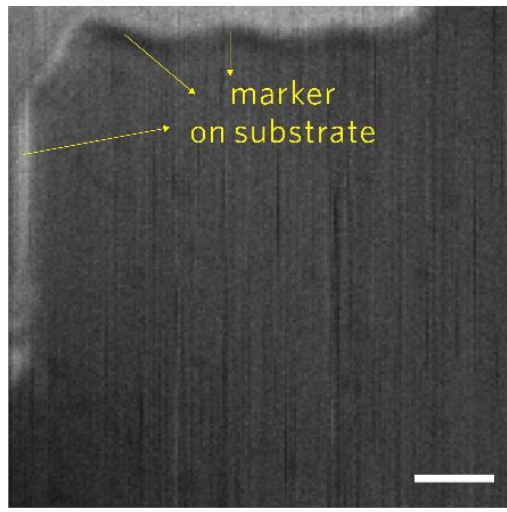

d SEM image

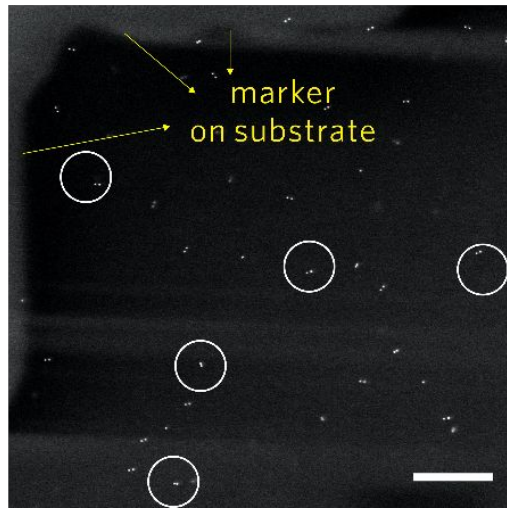

Figure S6. Determination of correlations between the emission polarization directions from the nanoclusters and the nanocluster structures. The Oct-QD-Au50-2 nanoclusters were deposited and dried on a silicon substrate with markers. The confocal FLIM image (a) and reflection image (b) were taken simultaneously on the single-molecule confocal microscopy. For typical single-molecule fluorescent clusters (white circles) in FLIM images, the PL intensity profiles as a functions of polarization directions were measured one by one and the polarization direction (major dipole direction) of each cluster was determined by its phase, as illustrated in c. With the guidance of the markers on the substrate, SEM image of the same area (d) were obtained to resolve the spatial configuration of each cluster. As shown in c and $d$, the emission polarization directions match well with the cluster axes. Note that lack of one-to-one correspondence originates from principal differences between the imaging modes of SEM and FLIM. In SEM, we could only see AuNPs, while only QDs could be detected in FLIM. Our Oct-QD-Au50-2 divalent clusters have a purity of around $75 \%$, and the impurity ( $25 \%$ correspondingly) leads to the clusters that might lack QD or AuNP and, consequently, to lack of one-to-one correspondence. Scale bars: $2 \mu \mathrm{m}$. 
a

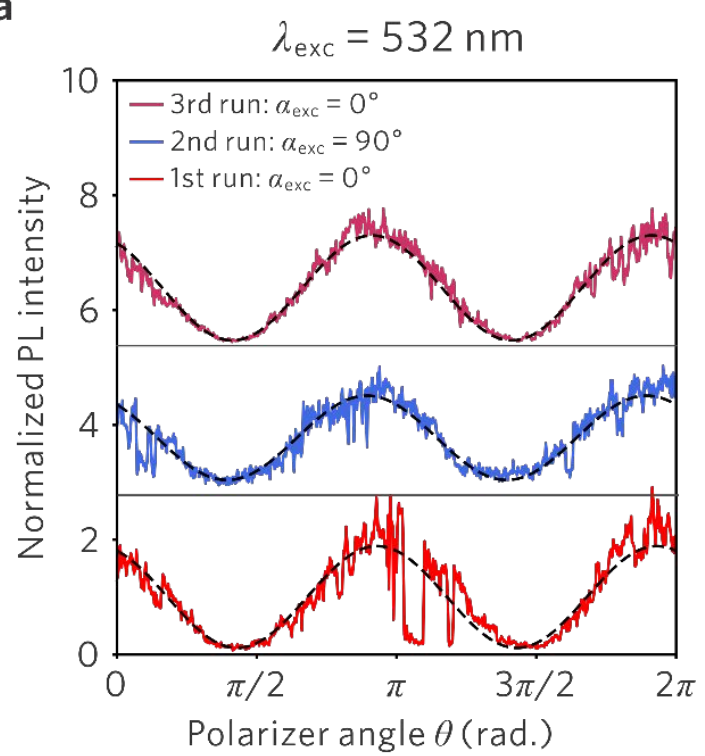

b

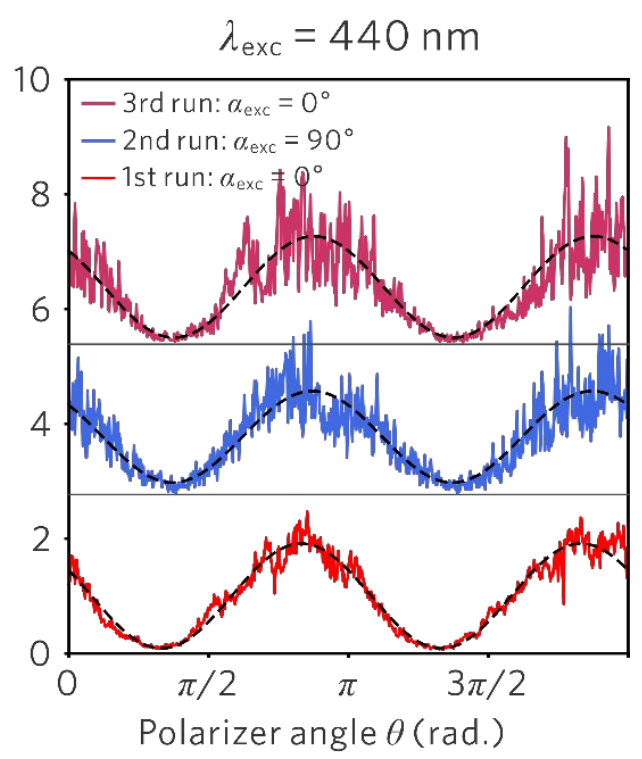

C

\begin{tabular}{lcccc}
\hline & \multicolumn{2}{c}{$\lambda_{\text {exc }}=532 \mathrm{~nm}$} & \multicolumn{2}{c}{$\lambda_{\text {exc }}=440 \mathrm{~nm}$} \\
\cline { 2 - 5 } & phase $\varphi$ & DOP of emission & phase $\varphi$ & DOP of emission \\
\hline 1st run: $\alpha_{\text {exc }}=0^{\circ}$ & $58^{\circ}$ & 0.89 & $75^{\circ}$ & 0.91 \\
2nd run: $\alpha_{\text {exc }}=90^{\circ}$ & $64^{\circ}$ & 0.73 & $68^{\circ}$ & 0.80 \\
3rd run: $\alpha_{\text {exc }}=0^{\circ}$ & $61^{\circ}$ & 0.91 & $67^{\circ}$ & 0.88 \\
\hline
\end{tabular}

Figure S7. Emission intensity profiles (as a function of emission polarization angles) at different fixed excitation polarization directions under optical excitation on SPR (a) and off SPR (b). For $\lambda_{\text {exc }}=532 \mathrm{~nm}$ and $440 \mathrm{~nm}$, the Oct-QD-Au50-2 nanoclusters were measured sequentially three times at different excitation polarization directions: $1^{\text {st }}$ run, $\alpha=0^{\circ} ; 2^{\text {nd }}$ run, $\alpha=90^{\circ} ; 3^{\text {rd }}$ run, $\alpha=0^{\circ}$. Little change was found for the emission profile phases as well as the DOP of emission (c) under both excitation wavelengths, which suggests that the emission polarization directions are independent on the excitation polarization directions. 

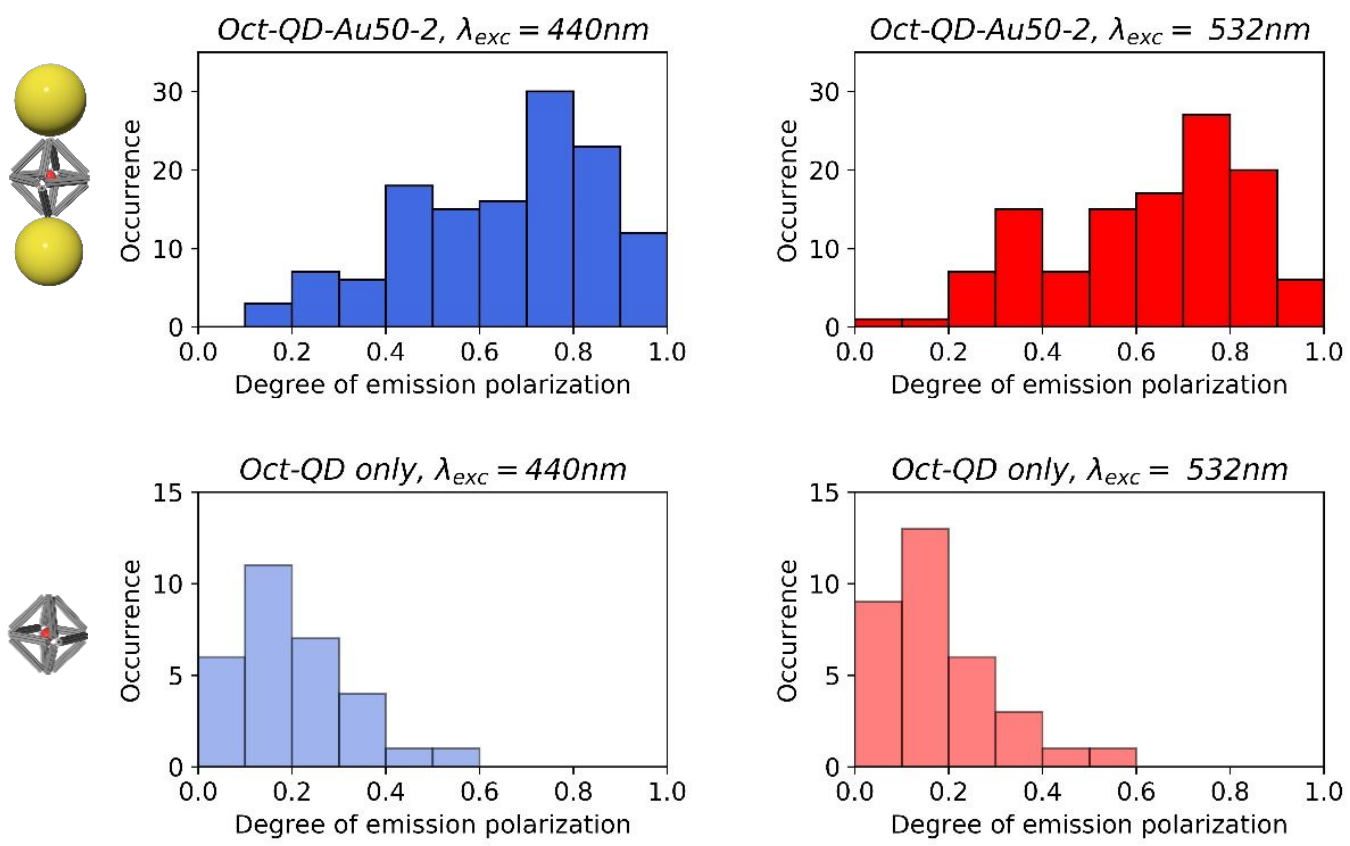

Figure S8. Histograms of degree of emission polarizations for Oct-QD-Au50-2 nanoclusters and Oct-QDs without AuNPs under optical excitation at $\lambda_{\text {exc }}=440$ and $532 \mathrm{~nm}$. Introducing AuNP dimers to the nanocluster systems greatly shifts the DOP to larger values. Different excitation wavelength does not show clear differences on the emission DOP distributions. 

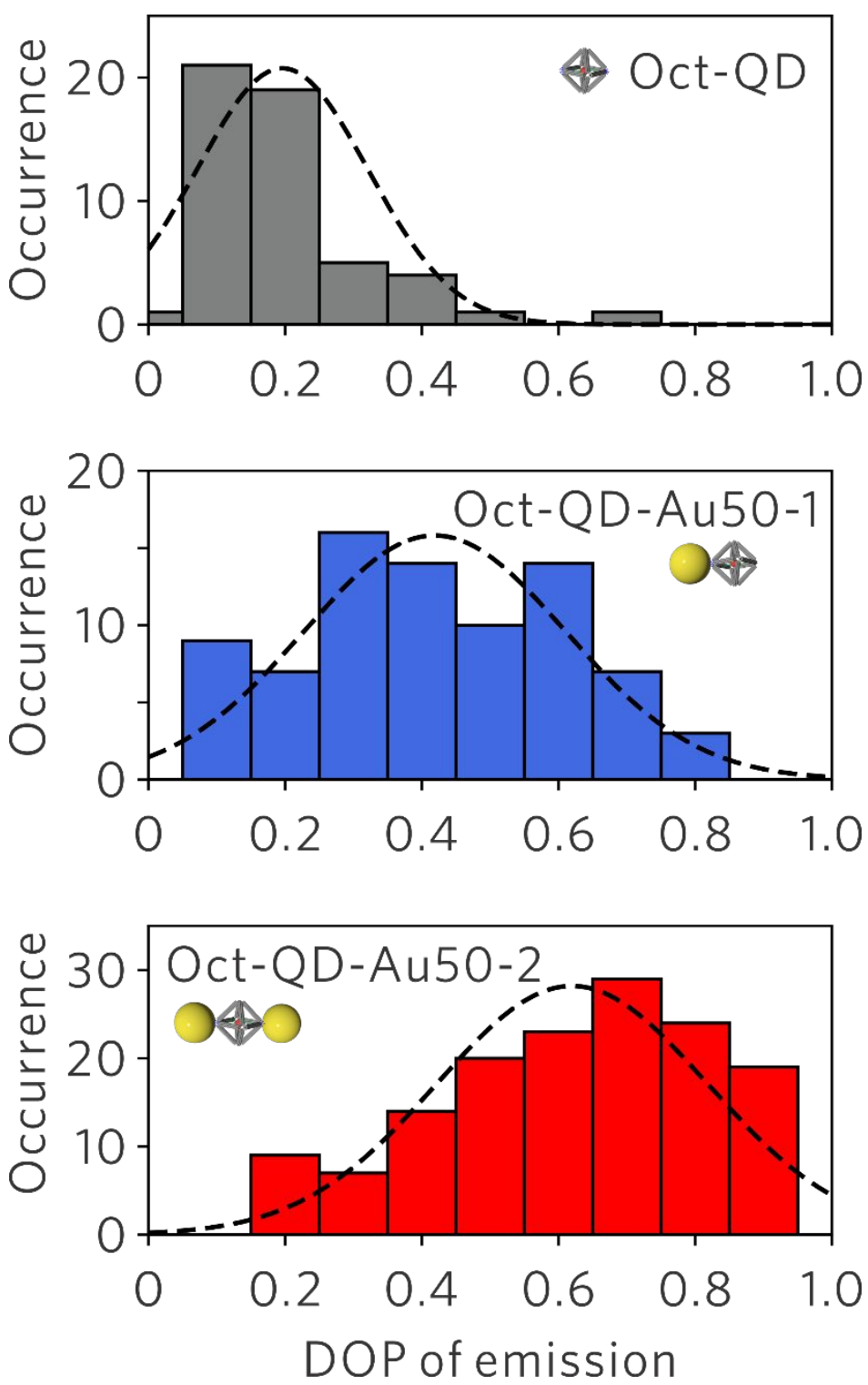

Figure S9. Histograms of degree of emission polarizations for Oct-QD, Oct-QD-Au50-1, Oct-QD-Au50-2 nanoclusters, respectively. The means DOP values are $\mathrm{DOP}_{\mathrm{QD}}=0.20 \pm 0.12, \mathrm{DOP}_{50-1}=0.42 \pm 0.19, \mathrm{DOP}_{50-2}$ $=0.62 \pm 0.20$. Theoretical calculations show that Oct-QD-Au50-1 and Oct-QD-Au50-2 nanoclusters exhibit emission DOP of 0.46 and 0.78 , which are consistent with the experimental results. 
a
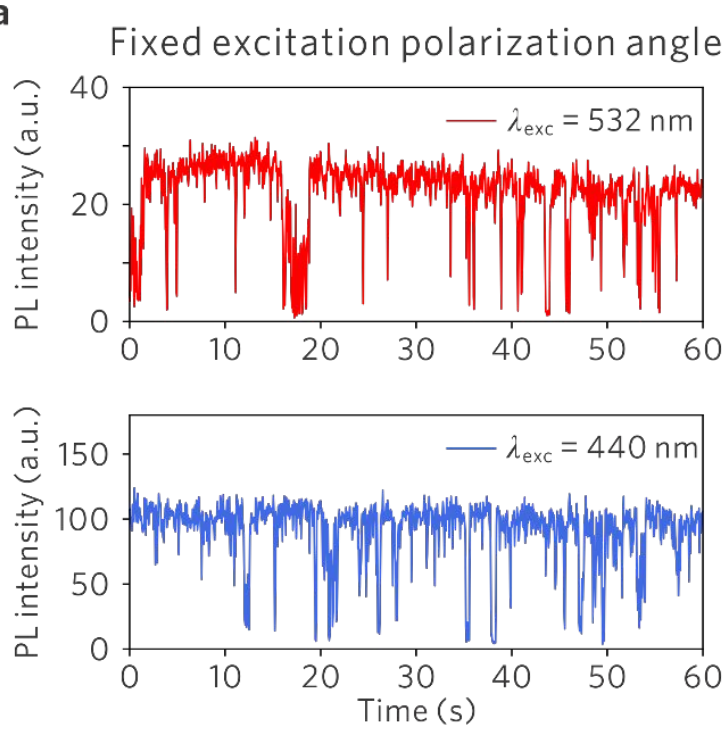

b
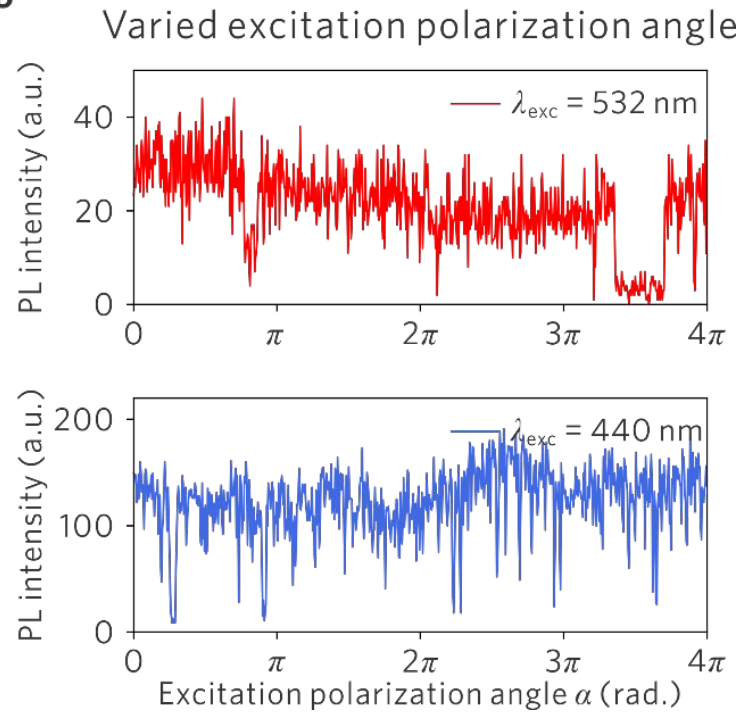

Figure S10. PL intensity profiles for the free QDs under different excitation conditions. a, Time trace of $P L$ intensity from a single $Q D$, under two optical excitation conditions ( $\lambda_{\text {exc }}=532$ and $440 \mathrm{~nm}$ ) at fixed an excitation polarization angle. $b, \mathrm{PL}$ intensities as a function of excitation polarization angles $\alpha$ for a single QD. No clear effect of excitation polarization on PL intensity modulation is found for free QDs.
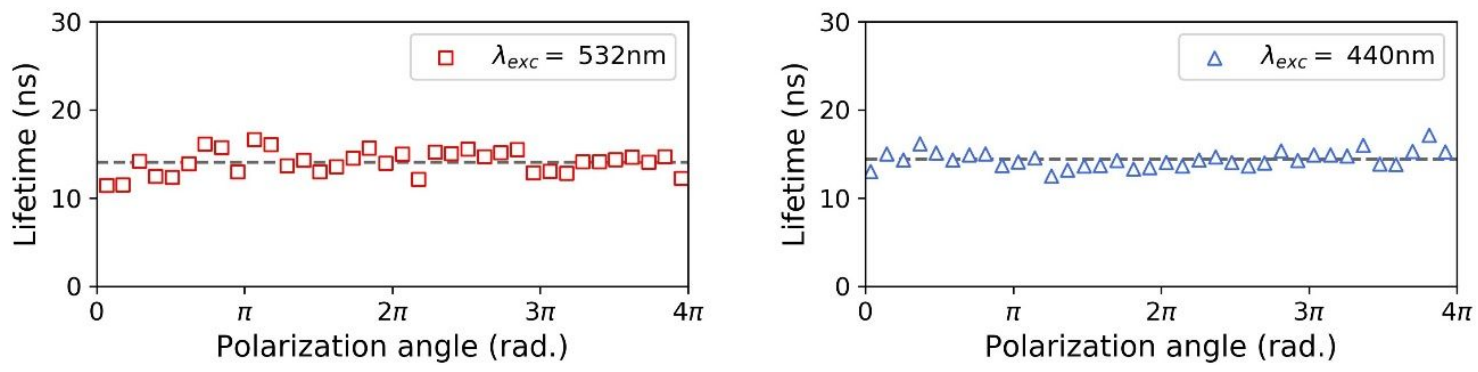

Figure S11. The lifetime as function of excitation polarization angles at the optical excitation of $532 \mathrm{~nm}$ and $440 \mathrm{~nm}$, corresponding to the PL intensity profiles in Fig. 3c. The lifetime at both wavelengths remains as a constant while excitation polarization angles is changed. 

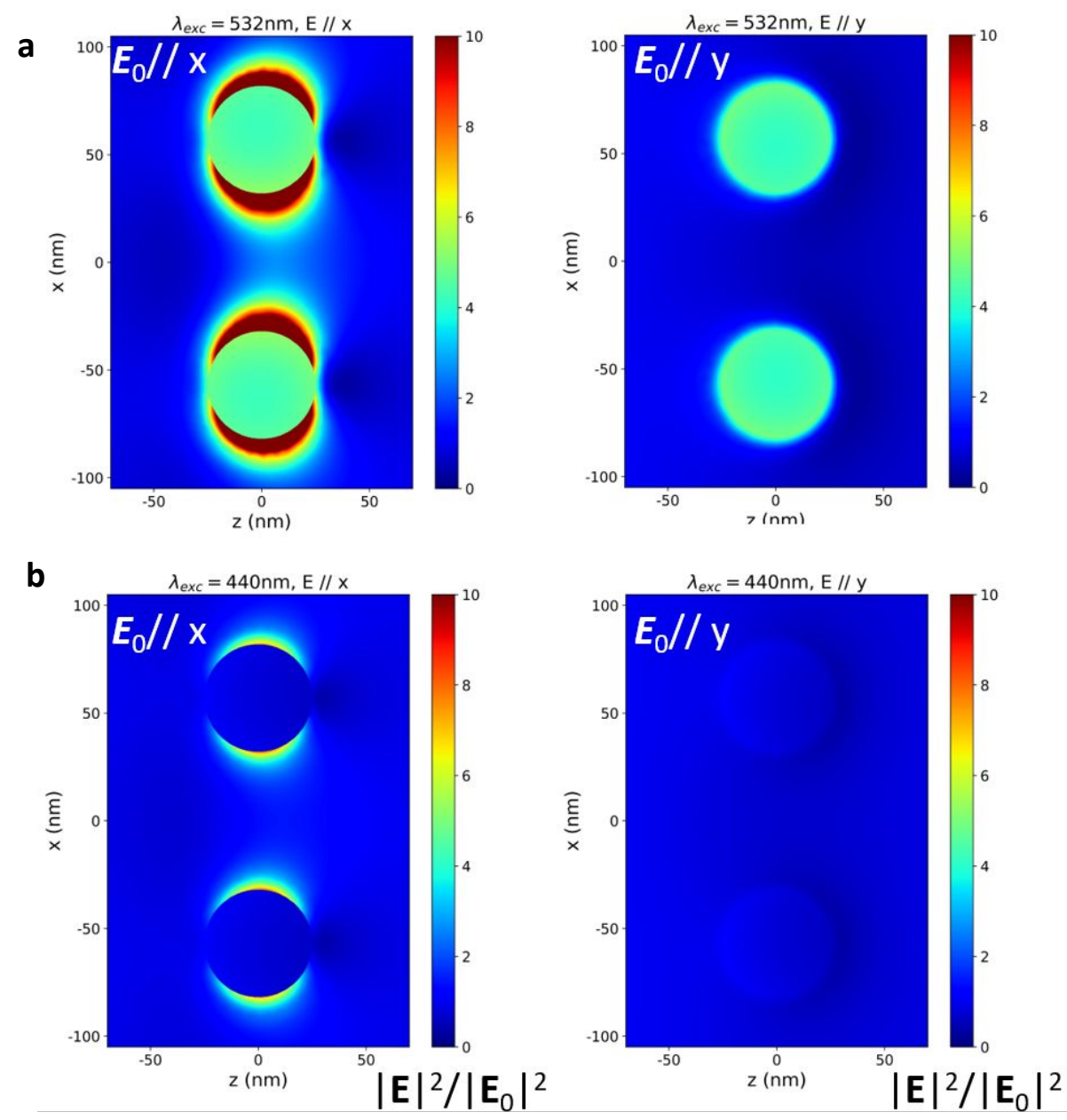

Figure S12. finite-element method (FEM)-simulated electric field enhancement $|E|^{2} /\left|E_{0}\right|^{2}$ for a AuNP dimer with the same structural parameters to the nanoclusters "Oct-QD-Au50-2" at $\lambda_{\text {exc }}=532 \mathrm{~nm}(\mathrm{a})$ and $440 \mathrm{~nm}$ (b) based on the Comsol simulation. The propagation of the field is along the $z$ axis and the polarization is along the $x$ and $y$ axes, respectively. 

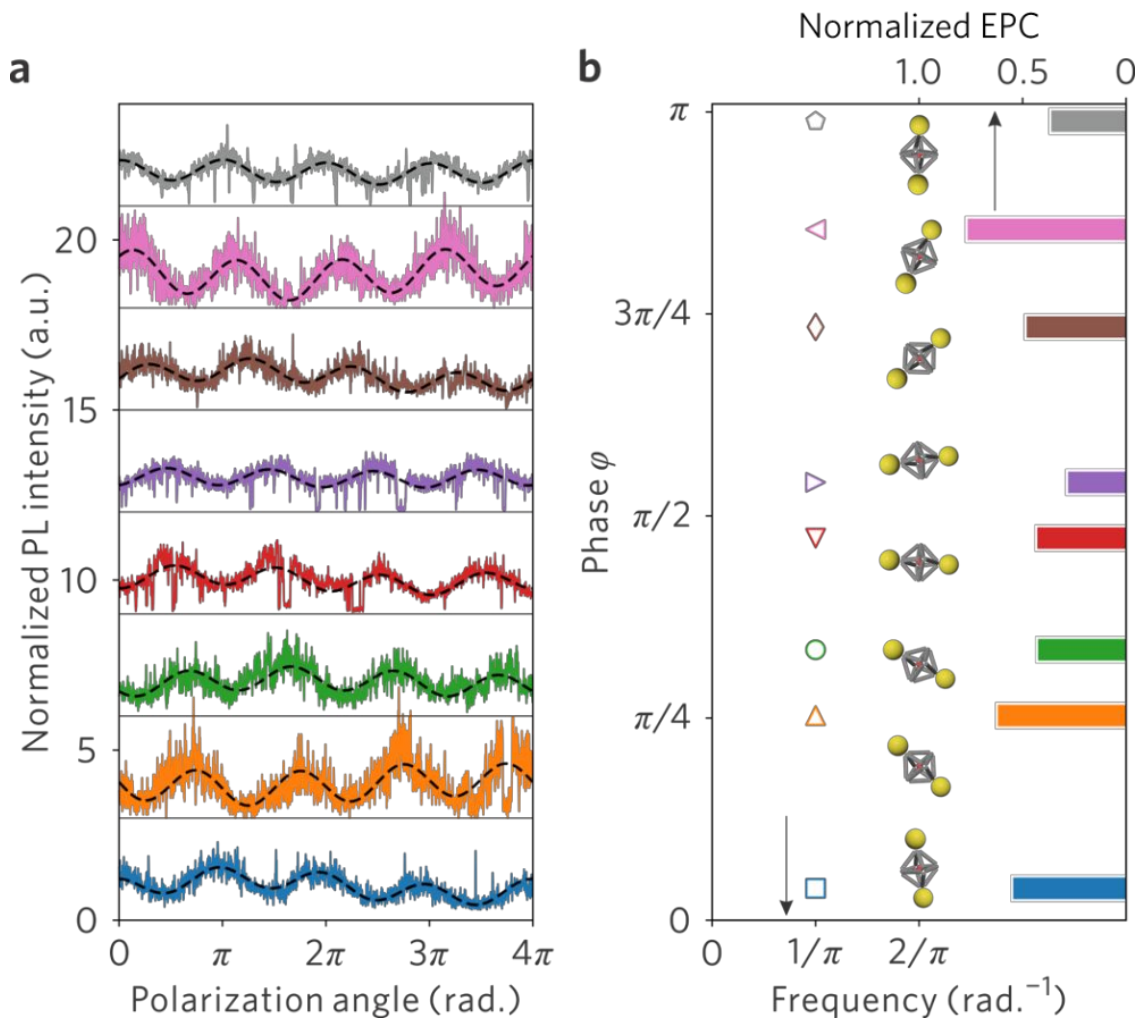

Figure S13. Phase shift from PL intensity profiles of a group of same-type nanoclusters with different spatial orientations under optical excitation at varied polarization angles. a, Normalized PL intensity from a group of nanoclusters, Oct-QD-Au50-2, excited at $\lambda_{\text {exc }}=532 \mathrm{~nm}$ under varied polarization angles. The plots are vertically shifted for clarity. $b$, Corresponding frequencies, phases and normalized EPC for the nanoclusters with PL profiles shown in a. Inset: Schematic of possible nanocluster configurations based on the extracted phases.
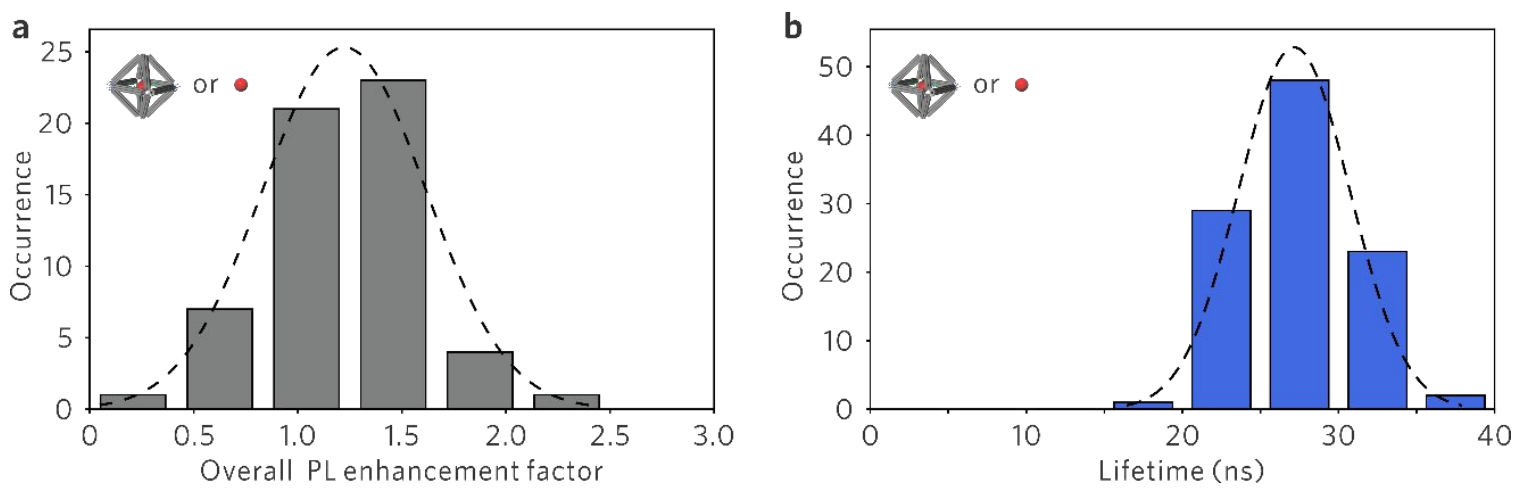

Figure S14. The histogram plots of overall PL enhancement factor (a) and lifetime (b) for individual QDs including free ssDNA-QDs and Oct-QDs. The black dash lines correspond to the normal distribution curves $\left(E F_{\mathrm{PL}}=1.2 \pm 0.4 ; \tau=27.3 \pm 3.6 \mathrm{~ns}\right)$. 

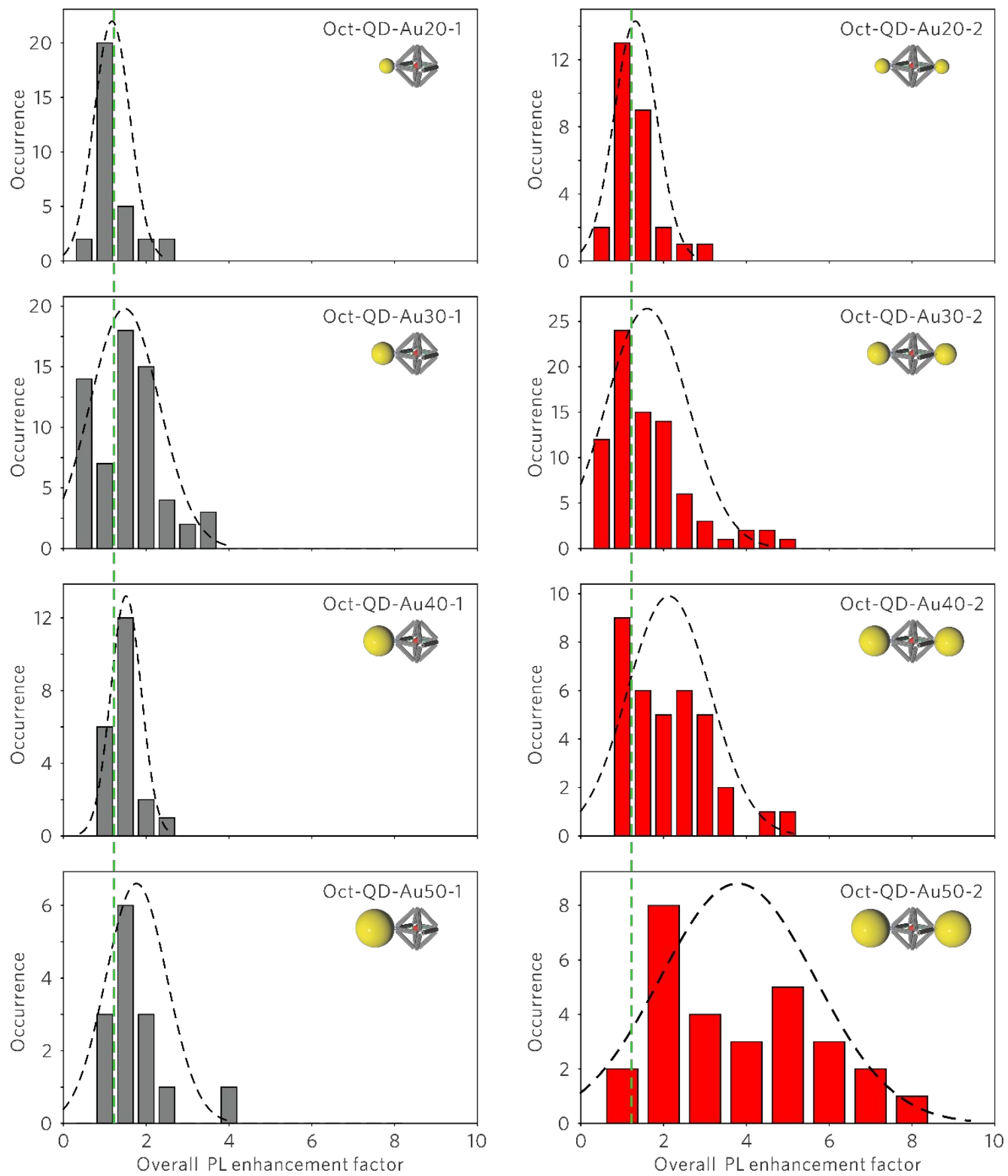

Figure S15. The histogram plots of PL enhancement factors for various Oct-QD-AuNP nanoclusters and the corresponding normal distribution curves (black dash). The green vertical dash lines represent the mean values of the over PL enhancement factor for QDs without AuNPs. 

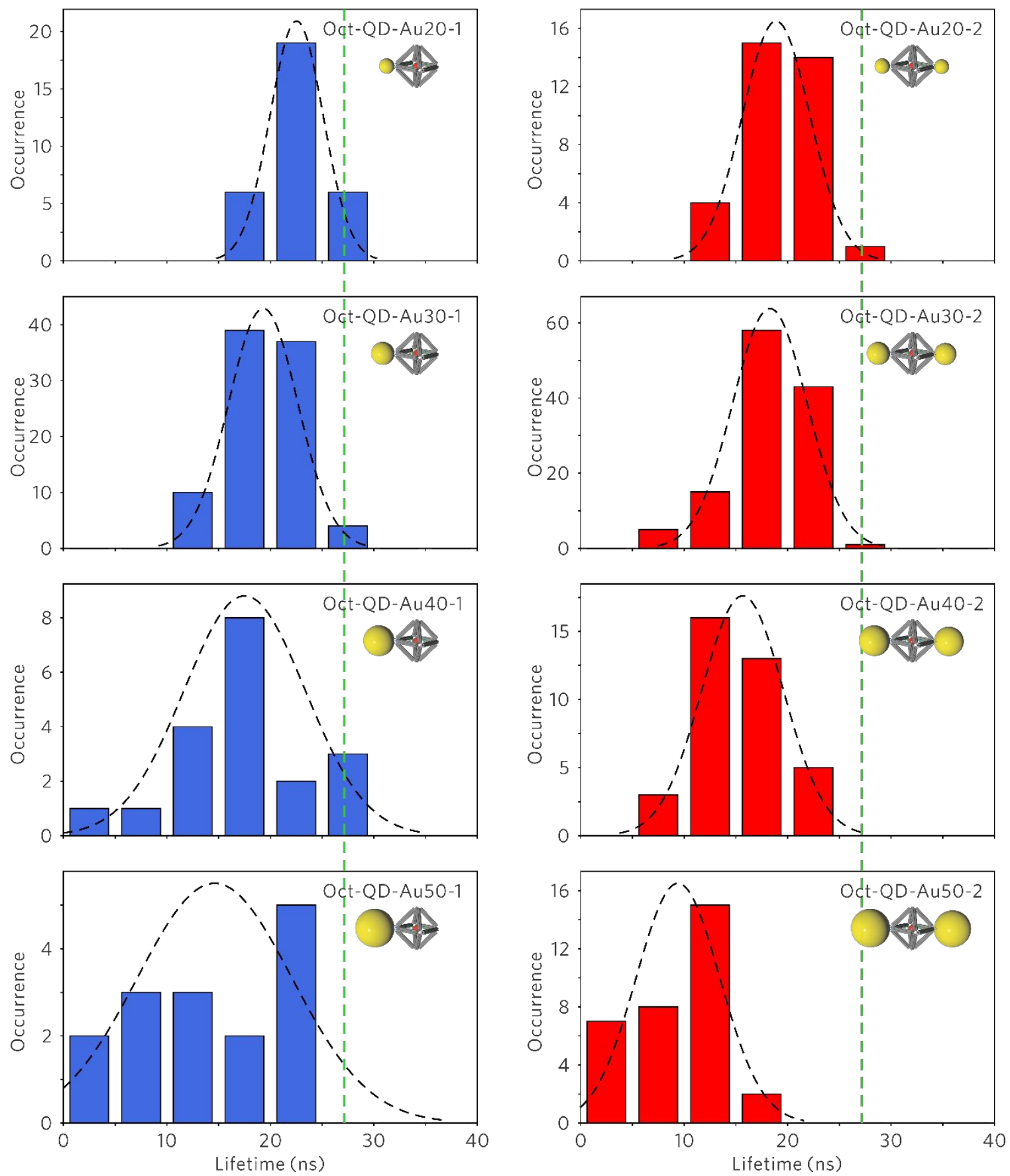

Figure S16. The histogram plots of lifetimes for various Oct-QD-AuNP nanoclusters and the corresponding normal distribution curves (black dash). The green vertical dash lines represent the mean values of the lifetime for QDs without AuNPs. 


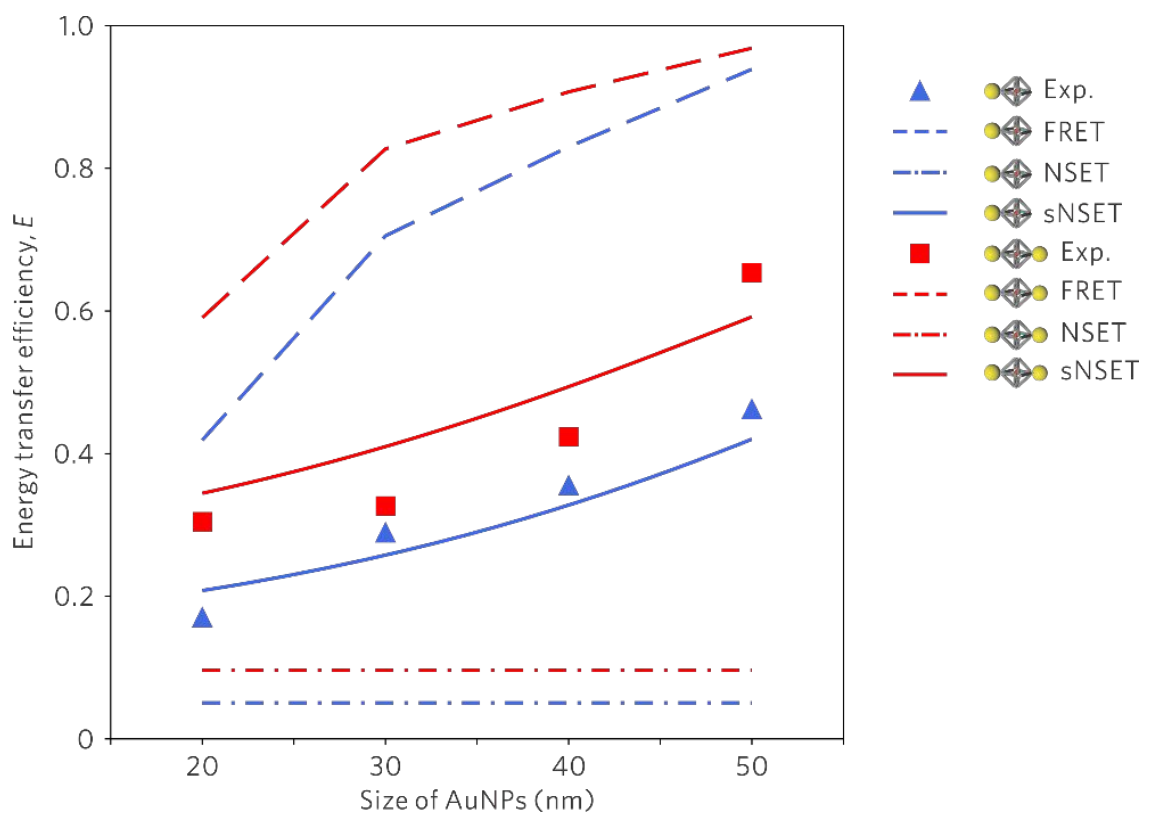

Figure S17. Comparison of various energy transfer models based on the experimental energy transfer efficiency, $E$. For the nanocluster systems with the AuNP monomers and dimers, the FRET model overestimates the $E$, while the traditional NSET model underestimates the $E$ and is size-independent. The modified NSET model (denoted SNSET) takes the size-dependent dielectric functions and absorbance of the AuNPs into account and shows a decent prediction of energy transfer in our nanocluster system (See more details in Energy Transfer section below). 
a

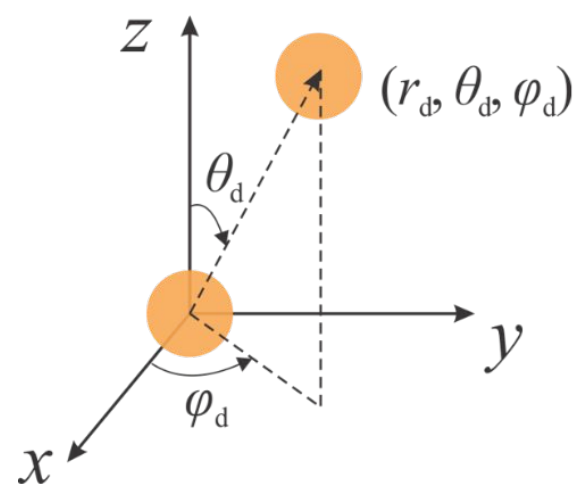

b

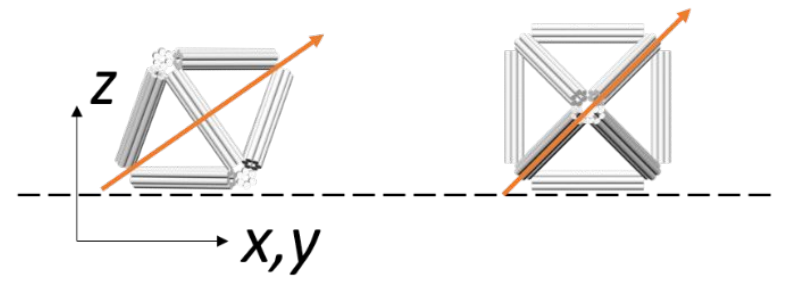

C

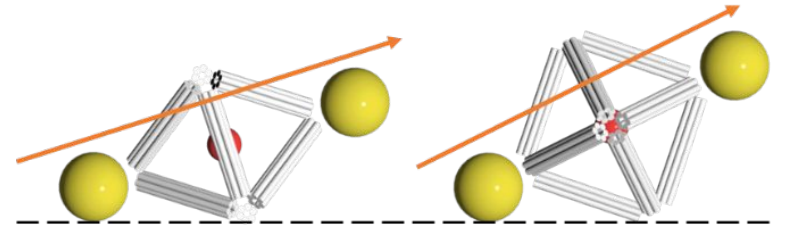

d

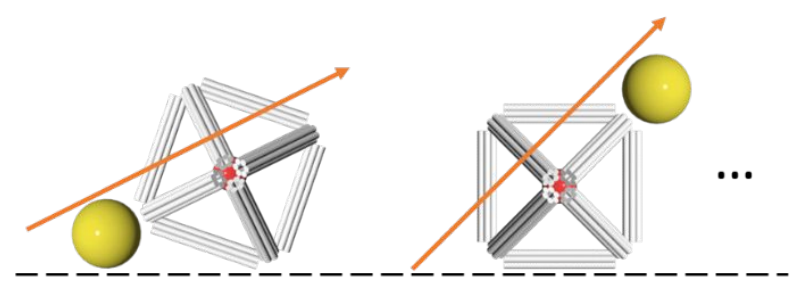

Figure S18. Spatial orientations of the nanoclusters at the liquid-solid interfaces during the confocal measurements of the excitation polarization effects. a, Schematic of a nanocluster orientation. $b$, Orientations of an octahedral DNA origami frame (arrows shown directions of two active vertices). c, Orientations of an Oct-QD-Au20-2 nanocluster. d, Representative orientations of an Oct-QD-Au20-1 nanocluster. We note that the directions of nanocluster axes with respect to the surface normal $\left(\theta_{d}\right)$ significantly affect the detection of the excitation polarization. The measured EPC values are the maximum EPC projected on the sample plane. When $\theta_{d}$ is very small, the excitation polarization becomes very difficult to detect (even if the nanocluster has large theoretical EPC). Thus, the nanoclusters show excitation polarization dependent detectable polar activity. Although the measured detectable polar activity shares similar trend with the simulated EPC (Fig. 4), it is uneasy to calculate the excitation polarization occurrence for the nanocluster systems because the spatial orientations of the nanoclusters are far from isotropic and impossible to use a simple model to predict. For instance, as shown in $b$, the origami frame itself has the $\theta_{d}$ limit (for one edge or one face touching the surfaces). When NPs are arranged in the frames, the spatial orientation distribution changes due to size effect of NPs and interactions between NPs and the surfaces. Moreover, the nanoclusters with dimers are symmetric and close to the pure frames (c), but the nanocluster with monomers are asymmetric and the scenarios of the orientation limits increase. In additions, the NPs with various sizes have different geometry constraints and interactions with the surfaces (such as van der Waals forces, electrostatic interactions), which makes the spatial orientation complicated. 
a

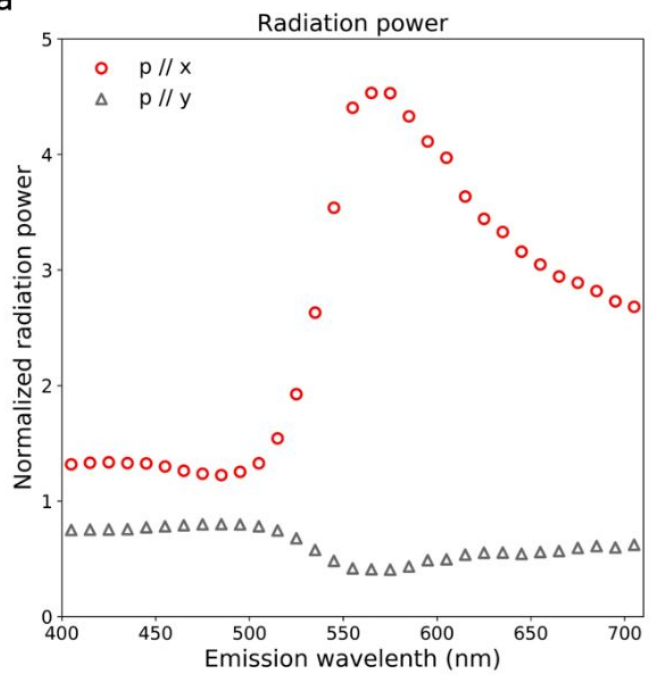

b

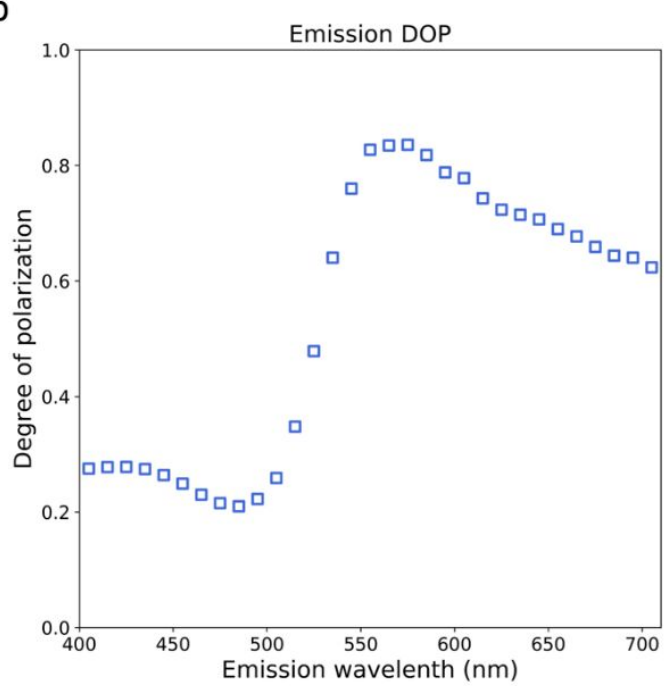

Figure S19. FEM-simulated emission polarization of a dipole moment in a divalent nanocluster with AuNP size of $50 \mathrm{~nm}$ with various levels of detuning between emission wavelengths and surface plasmon of AuNPs. a, Normalized radiation power of a point dipole moment with different emission wavelengths and with its vector parallel $(\boldsymbol{p} / / \mathrm{x})$ and perpendicular $(\boldsymbol{p} / / \mathrm{y})$ to the nanocluster axis (x axis). b, DOP of radiation power as function of the emission wavelengths.

a

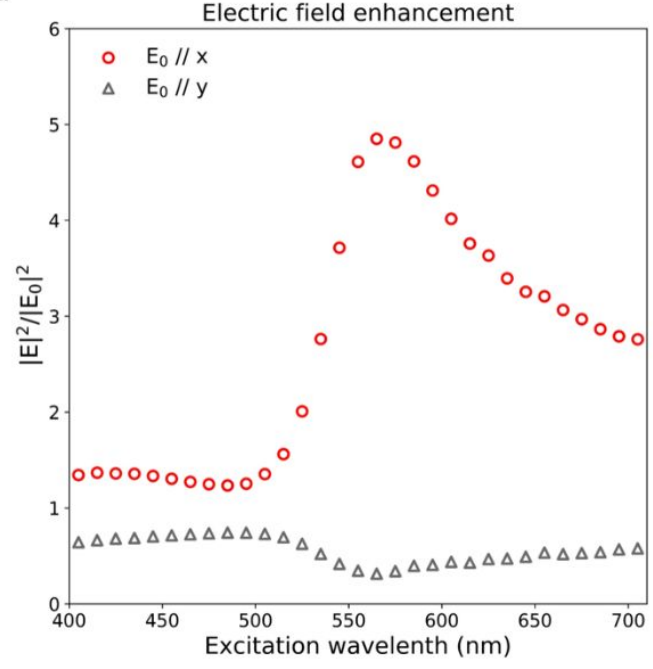

$\mathrm{b}$

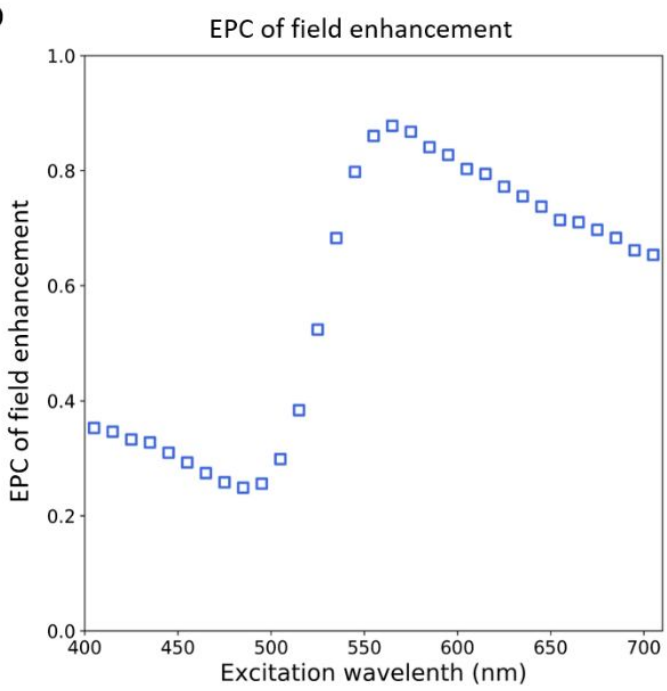

Figure S20. FEM-simulated electric field enhancement and EPC of field enhancement of a divalent nanocluster with AuNP size of $50 \mathrm{~nm}$ with various excitation photon energies. a, Electric field enhancement in the position of the QD for excitation polarization direction parallel $\left(E_{0} / / x\right)$ and perpendicular $\left(E_{0} / / y\right)$ to the nanocluster axis ( $x$ axis). b, EPC of field enhancement as a function of the excitation wavelengths. Excitation around SPR increases the EPC of the field enhancement. 


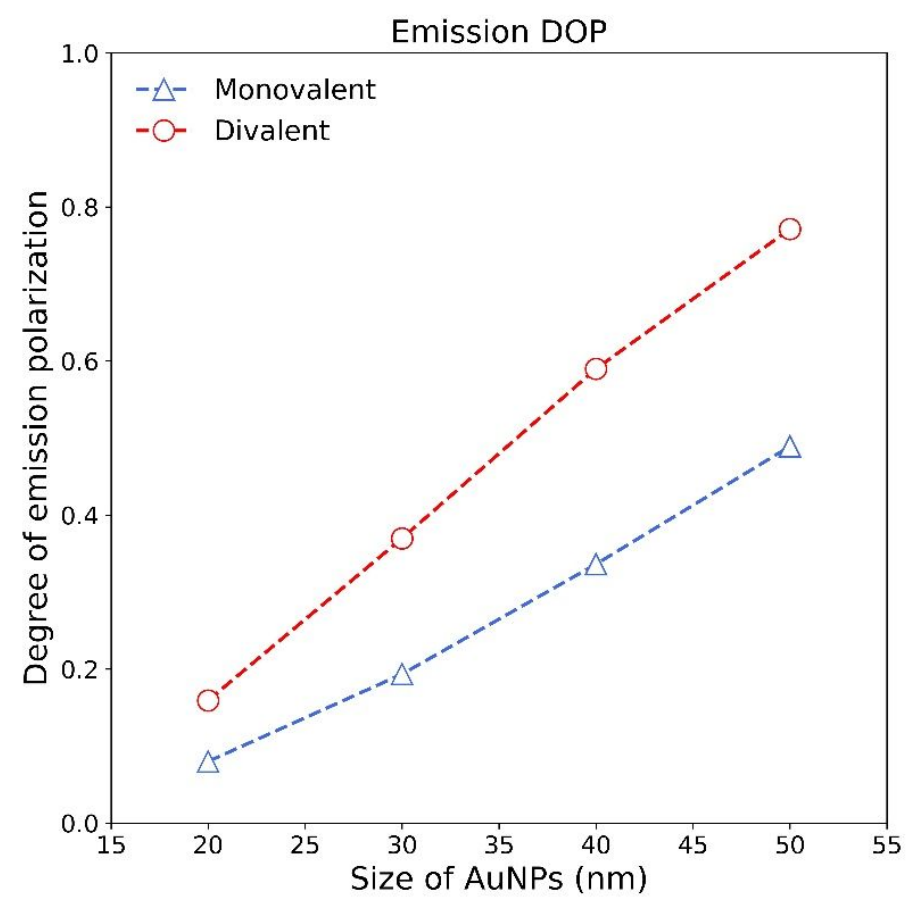

Figure S21. FEM-simulated emission polarization of a dipole moment $\left(\lambda_{\text {emi }}=605 \mathrm{~nm}\right)$ in a mono- and divalent nanocluster with AuNP size of $20-50 \mathrm{~nm}$. 


\section{Experimental methods}

Materials. The DNA scaffold strands, M13mp18 single-stranded phage DNA, were purchased from Bayou Biolabs. Unmodified staple strands and outer linker staple strands were purchased from Integrated DNA Technologies, Inc. (IDT) in RNase-free water. Inner anchoring staple strands and biotinylated singlestranded DNA (ssDNA) were purchased as lyophilized powers from IDT and were purified with polyacrylamide gel electrophoresis (PAGE) by the manufacturer. Thiolated ssDNA, purified using high performance liquid chromatography (HPLC), were purchased as lyophilized powers from IDT. All the DNA strands were used as received without further purification. Streptavidin-conjugated quantum dots (QDs) with the emission maxima of $\sim 605 \mathrm{~nm}$ were purchased from Thermo Fisher Scientific Inc. Citrate-stabilized gold nanoparticles (AuNPs) with the nominal diameters of 20, 30, 40 and $50 \mathrm{~nm}$ were purchased from Ted Pella, Inc.

DNA functionalization of AuNPs. The thiolated ssDNA was mixed with tris(2-carboxyethyl)phosphine (TCEP) aqueous solution (Sigma-Aldrich) in a molar ratio of DNA/TCEP $=1: 100$ and incubated at room temperature for 1.5 hours to cleave the disulfide bond. The cleaved thiolated ssDNA was purified with a size exclusion column (MicroSpin G-25, GE Healthcare). The purified thiolated ssDNA was then mixed with aqueous AuNP solution in a chosen molar ratio (for 20,30, 40 and $50 \mathrm{~nm}$ AuNPs, the molar ratio of DNA/AuNP $=1000: 1,2100: 1,3600: 1$ and 5500:1, respectively). After $2 \mathrm{~h}$ of incubation under orbital shaking, the mixture was buffered with a $10 \times$ phosphate buffer ( $10 \times \mathrm{PB}, 100 \mathrm{mM}, \mathrm{pH} 7.4)$ to obtain a final phosphate concentration of $10 \mathrm{mM}$. Meanwhile, $10 \%$ (wt/vol) sodium dodecyl sulfate (SDS) solution was added and the final SDS concentration was brought up to $\sim 0.01 \%$. After another $2 \mathrm{~h}$ of incubation, a salting process was performed. Specifically, phosphate-buffered saline (PBS, $10 \mathrm{mM}$ phosphate, $2.0 \mathrm{M} \mathrm{NaCl}, \mathrm{pH}$ 7.4) was gradually added to the mixture to increase the $\mathrm{NaCl}$ concentration stepwise from 0 to $0.3 \mathrm{M}$ over six steps. Each step consists of 20-s vortexing, 20-s sonication and 1-h incubation at room temperature. The final mixture was aged at room temperature under orbital shaking for at least half day before washing. The AuNPs functionalized with ssDNA were purified by at least 4 cycles of centrifugation to remove excess unbound DNA. In each cycle, the supernatant was carefully discarded and the AuNP pellets at the bottom of the tubes were resuspended in a PBS buffer ( $10 \mathrm{mM}$ phosphate, $100 \mathrm{mM} \mathrm{NaCl}, 0.01 \% \mathrm{SDS}, \mathrm{pH}$ 7.4). For the last cycle of centrifugation, the pellets were resuspended in $1 \times \mathrm{TAE} / \mathrm{Mg}^{2+}$ buffer ( $40 \mathrm{mM}$ Tris acetate, $1 \mathrm{mM}$ EDTA, $12.5 \mathrm{mM}$ Magnesium acetate, $\mathrm{pH}$ 8.3). The final DNA-functionalized AuNPs (DNA-AuNPs) were redispersed in $1 \times \mathrm{TAE} / \mathrm{Mg}^{2+}$ buffer and the concentrations were determined by ultraviolet-visible (UV-vis) absorption obtained from a UV-vis spectrophotometer (LAMBDA 25, PerkinElmer).

DNA functionalization of QDs. The biotinylated ssDNA was mixed with the streptavidin-conjugated QDs in a molar ratio of ssDNA/QD = 10-15:1. The mixture was buffered to $1 \times \mathrm{TAE} / \mathrm{Mg}^{2+}$ using a $10 \times \mathrm{TAE} / \mathrm{Mg}^{2+}$ buffer solution. The mixture was wrapped with aluminum foil and incubated at room temperature for half a day, prior to storage at $4{ }^{\circ} \mathrm{C}$. It is worth noting that most of the biotinylated ssDNA was bound to QDs as each QD comprises approximately 5-10 streptavidin molecules and each streptavidin molecule has 4 binding sites for biotin with a high affinity. Thus, the purification step for as-prepared DNA-QDs was skipped.

Assembly of octahedral DNA origami. The molecular structure of the octahedral DNA origami frame was adapted from our previous design ${ }^{1}$ using caDNAno software. The DNA octahedron is constructed with twelve edges, one of which is a six-helix bundle (6HB). One vertex or two opposite vertices contain four dangling ssDNA (outer linker staple strands) which is active for binding of DNA-AuNPs. Selected four in- 
plane edges have eight ssDNA (inner anchoring staple strands) projecting toward the interior of the octahedron to encapsulate DNA-QDs. The octahedral DNA origami was prepared using a thermal annealing process. Briefly, the M13mp18 scaffold DNA was mixed with unmodified staple strands, outer linker strands and inner anchoring strands in a molar ratio of 1:10:10:10. The mixture was brought up to a final volume of $100 \mu \mathrm{L}$ with $10 \mathrm{nM}$ scaffold DNA in $1 \times \mathrm{TAE} / \mathrm{Mg}^{2+}$ buffer. The annealing was carried out in a thermal cycler (Mastercycler, Eppendorf), where the mixture was rapidly heated up to $90{ }^{\circ} \mathrm{C}$ and then slowly cooled down to $16^{\circ} \mathrm{C}$ over the course of $23 \mathrm{~h}$.

Fabrication of binary nanoparticle clusters. The octahedral DNA origami (Oct) was mixed with DNA-QDs in a molar ratio of Oct/QD $=1: 10$ in $1 \times \mathrm{TAE} / \mathrm{Mg}^{2+}$ buffer. During the annealing process, the mixture was heated to $50{ }^{\circ} \mathrm{C}$ within $2 \mathrm{~min}$ and hold at $50{ }^{\circ} \mathrm{C}$ for $2 \mathrm{~h}$, following by cooling down to $15^{\circ} \mathrm{C}$ over $\sim 1 \mathrm{~h}$. The as-prepared octahedron-caged QDs were directly mixed with DNA-AuNPs (also in $1 \times \mathrm{TAE} / \mathrm{Mg}^{2+}$ buffer) in a selected molar ratio of active octahedral vertices (Vtx) to AuNPs (for 20 and $30 \mathrm{~nm}$ AuNPs, the molar ratio of $\mathrm{Vtx} / \mathrm{AuNP}=1: 10$; for 40 and $50 \mathrm{~nm}$ AuNPs, the molar ratio of $\mathrm{Vtx} / \mathrm{AuNP}=1: 5)$. The mixture was aged at $45^{\circ} \mathrm{C}$ for $5 \mathrm{~min}$ and cooled down to $20^{\circ} \mathrm{C}$ over $2 \mathrm{~h}$. To remove the excess QDs and AuNPs, the final product was purified by $0.8-0.9 \%$ agarose gel in $1 \times$ TBE buffer ( $89 \mathrm{mM}$ Tris borate, $2 \mathrm{mM}$ EDTA, pH 8.3) containing $10 \mathrm{mM} \mathrm{MgCl}_{2}$. The gel electrophoresis was performed at $60 \mathrm{~V}$ for 3-5 $\mathrm{h}$ in a horizontal gel system (Owl EasyCast, Thermo Scientific) in an ice bath. The band of target DNA origami-AuNP clusters was cut and chopped into pieces using a clean razor blade. The nanoclusters were recovered from the gel pieces using a gel extraction column (Freeze 'N Squeeze, Bio-Rad). 
a

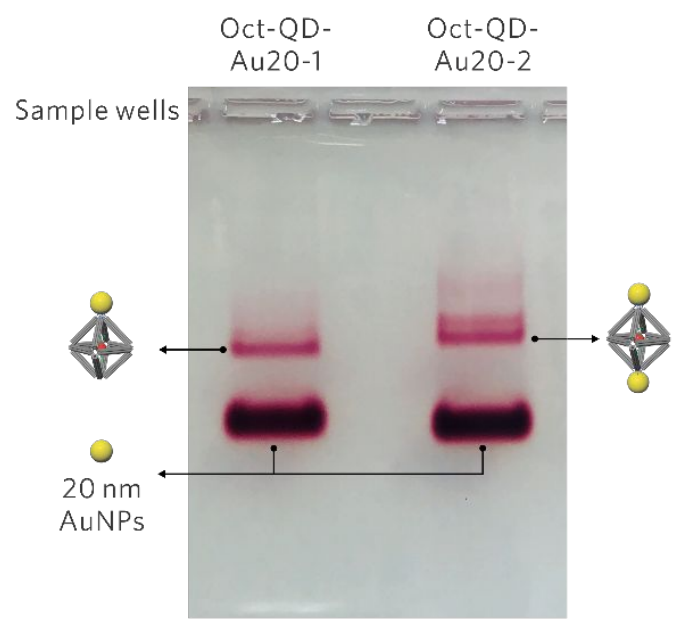

C
Oct-QD- Oct-QD- Oct-QD- Oct-QD-
Au20-2 Au30-2 Au40-2 Au50-2

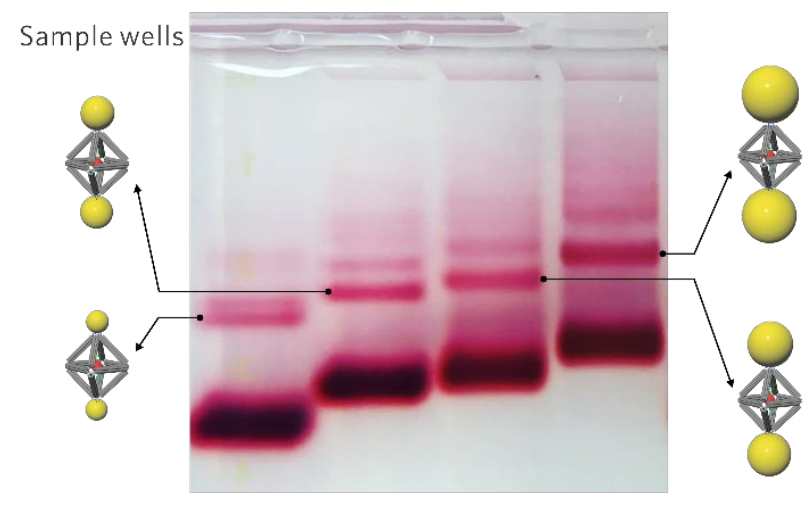

b

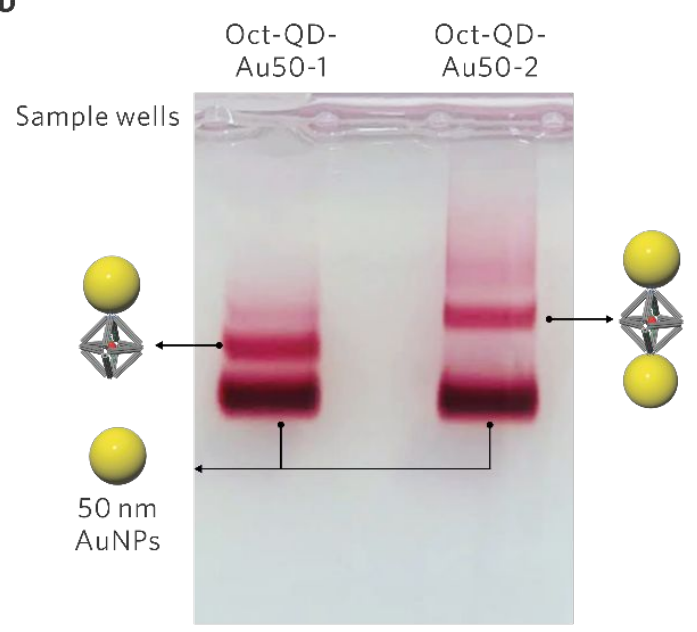

d

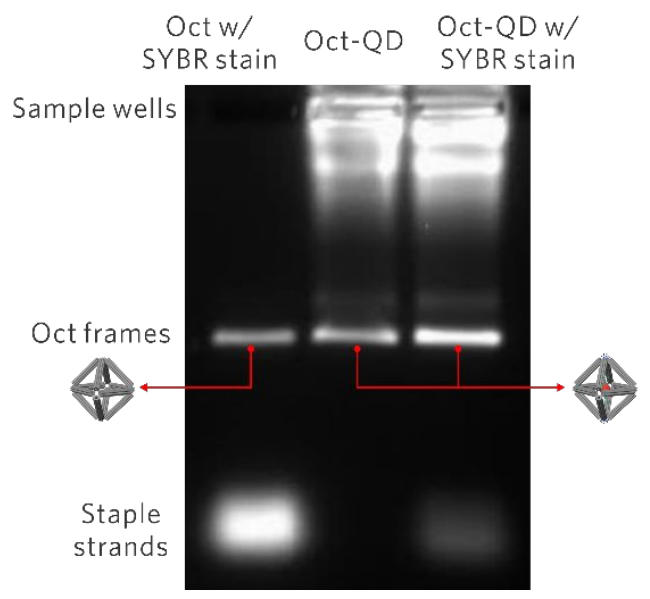

Figure S22. Gel electrophoresis images. a, A white light image of mono- and di-valent nanoclusters with 20-nm-sized AuNPs. b, A white light image of mono- and di-valent nanoclusters with 50-nm-sized AuNPs. c, A white light image of divalent nanoclusters with AuNPs of various sizes (20,30, 40 and $50 \mathrm{~nm}$ ). d, A UV light image of empty octahedron frames (Oct) with the SYBR gold stain and Oct-caged QDs (Oct-QD) with and without the SYBR gold stain. 


\section{Structural characterizations}

Transmission Electron Microscopy (TEM). An aliquot (typically $5 \mu \mathrm{L}$ ) of the aqueous suspension nanocluster sample was deposited on the carbon-coated copper grid (300 mesh, Ted Pella). After incubation for $5 \mathrm{~min}$, excess solution was removed by filter paper. The grid was rinsed with water $(5 \mu \mathrm{L})$ twice. Then, the negative staining was performed by depositing $5 \mu \mathrm{L}$ of $2 \%$ uranyl acetate solution on the grid and staining for $15 \mathrm{~s}$. After staining, the sample was imaged on a JEM-1400 TEM (JEOL) operating at $120 \mathrm{kV}$.

Small Angle X-ray Scattering (SAXS). SAXS measurements of divalent nanoclusters in buffer solution (1X TBE, $10 \mathrm{mM} \mathrm{MgCl}$ ) were conducted at the Complex Materials Scattering (CMS) beamline at the National Synchrotron Light Source II (NSLS-II). The collected scattering 2D images were converted to 1D scattering intensity profiles I(q) as a function of the scattering vector magnitude $q=4 \pi \sin (\theta) / \lambda$ with $2 \theta$ being the scattering angle and $\lambda$ being incident $X$-ray wavelength. The freely dispersed DNA-capped AuNPs were measured to obtain the form factor $\mathrm{P}(\mathrm{q})$ of AuNPs. Scattering from the pure buffer solution was used as background. The structure factor $S(q)$ of the divalent nanoclusters was calculated by $S(q)=I(q) / P(q)$ after background subtraction. The scattering profiles were fitted as a dumbbell model with $S(q)=\sin (q D) /(q D)$ +1 , where $D$ is the center-to-center distance of the two particles. Here, $D$ is assumed to obey a Gaussian distribution $\mathrm{f}(\mathrm{D})$ with $\int_{0}^{\infty} f(D) d D=1$. Thus, $S(q) \propto \int_{0}^{\infty}\left(\frac{\sin (q D)}{q D}+1\right) f(D) d D$.

Scanning Electron Microscopy (SEM). The cluster sample deposited on the silicon substrate (with markers) was imaged on a Hitachi 4800 SEM operating at $5 \mathrm{kV}$, after optical characterizations. 


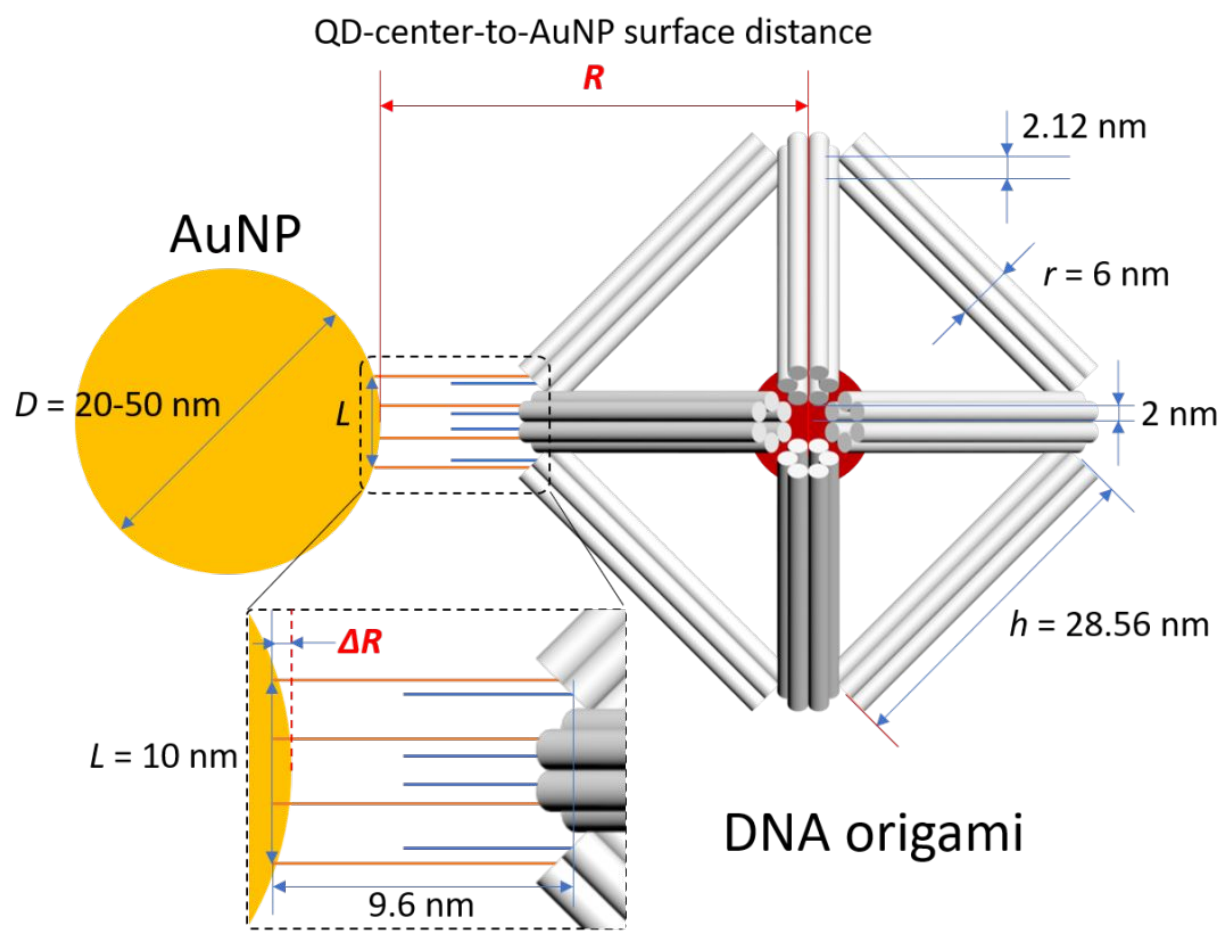

Figure S23. Geometry of the Oct-QD-AuNP nanoclusters. The footprint size of an octahedral origami vertex on the AuNPs is around $10 \mathrm{~nm}(L=10 \mathrm{~nm})$, and it is assumed to be independent of AuNP sizes. The QD-center-to-AuNP surface distance $R=33.9-\Delta R(\mathrm{~nm})$. Since $\Delta R=\frac{D}{2}-\sqrt{\left(\frac{D}{2}\right)^{2}-\left(\frac{L}{2}\right)^{2}}, \Delta R$ decreases from 1.3 to $0.5 \mathrm{~nm}$ when $D$ increases from 20 to $50 \mathrm{~nm}$. The $R$ increases with the size of the AuNPs increased, which is consistent with the results from $X$-ray scattering. 


\section{Optical characterizations}

Fluorescence spectroscopy. The photoluminescence spectrum of the QDs was measured using the Cary Eclipse fluorescence spectrophotometer (Varian) with the excitation wavelength of $400 \mathrm{~nm}$.

Single-molecule confocal microscopy. Time-resolved confocal fluorescence-lifetime imaging microscopy (FLIM) measurements were performed on a home-built inverted microscope (Olympus IX81) with raster scanning stage mounted with a 60x, 1.2 NA water immersion objective. The sample was excited by a pulsed diode-pumped solid-state laser system (LHD-440 PicoQuant, wavelength 440 nm; LHD-532 PicoQuant, wavelength $532 \mathrm{~nm}$; full width at half maximum $90 \mathrm{ps}$ ) operated at $10 \mathrm{MHz}$ repetition rate with an average power of $300-500 \mathrm{nW}$. The power ratio of $P_{532 \mathrm{~nm}} / P_{440 \mathrm{~nm}}$ was kept as 1.2 to account for differences in photon energy. The photoluminescence $(\mathrm{PL})$ was collected in the epi-illumination scheme, spectrally separated from the excitation laser light by a dichroic mirror (Semrock, DiO-532) and spatially filtered by a $100 \mu \mathrm{m}$ pinhole and a bandpass filter (HQ 605/50). The PL was imaged onto the single-photoncounting avalanche photodiodes (MPD PicoQuant) coupled to a time-analyzer (PicoHarp 300, PicoQuant). Typical setups were presented below to detect the emission polarization and the effect of excitation polarization. Data acquisition and data analysis were performed with the Symphotime 64 analysis software (PicoQuant).

\subsection{Single-molecule PL and lifetime measurements}

For single-molecule PL and lifetime measurements, the sample solutions (typically $0.8 \mu \mathrm{L}$ with the concentration in tens to hundreds of picomolar range) were sandwiched by hydrophobic coverslips. The thickness of the liquid films between the coverslips was 1-4 microns determined by focusing the upper and lower liquid-solid interfaces under the optical microscope. During the measurement, the focus was set to the lower liquid solid interface. As shown in Fig. S24, each sample point was excited at two wavelengths $\left(\lambda_{\mathrm{exc}}=532\right.$ and $440 \mathrm{~nm}$ ) and the PL was filtered by a bandpass filter (HQ605/50).

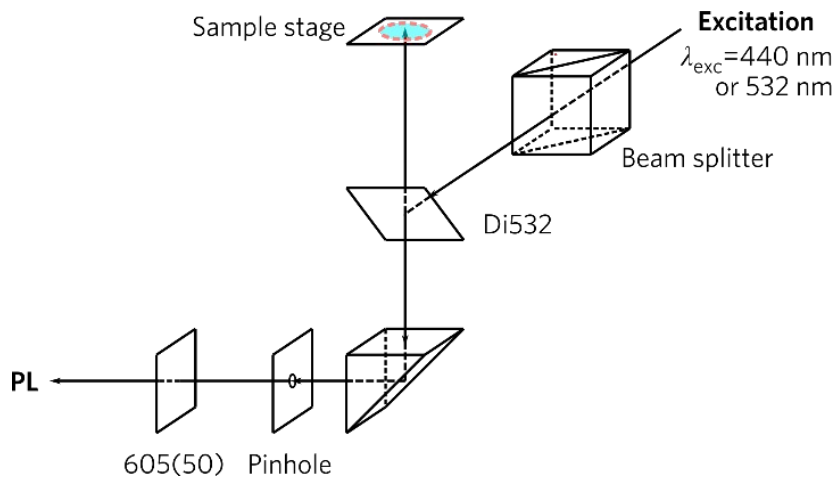

Figure S24. The instrumental setup for detection of PL and lifetime from individual light-emitting nanoclusters. For the same nanocluster, the PL intensity at two excitation wavelengths $\left(\lambda_{\mathrm{exc}}=532\right.$ and 440 $\mathrm{nm}$ ) are collected.

\subsection{Emission polarization detection}

The sandwiched liquid samples, typically the Oct-QD and the Oct-QD-Au50-2, were used to measure the emission polarization and emission DOP distributions. A linear polarizer was inserted in front the detector. 
By rotating the polarizer with a speed of $10 \%$, the PL intensity profiles as a function of the polarizer angles $\theta$ were collected (Fig. S25). The phases of the PL intensity profiles were extracted to determine the emission polarization directions.

In order to justify the correlation between the emission polarization directions and the nanocluster configurations, the Oct-QD-Au50-2 nanoclusters were deposited on the silicon substrate with markers fabricated by chemical vapor deposition and dried before the confocal measurements. As shown in Fig. S25, the FLIM image and reflection image were collected simultaneously by using a dichroic mirror (Semrock, DiO-442) and exciting at $440 \mathrm{~nm}$ (See typical images in Fig. S5). It is worth noting that during image taken, the polarizer was removed to avoid photoselections. After the image taken, the polarizer was mounted to determine the emission polarization profiles for selected fluorescent points (Fig. S5). The markers were used to track the nanocluster position under SEM and bridge the coordination between the FLIM images and SEM images (See typical images in Fig. S6).

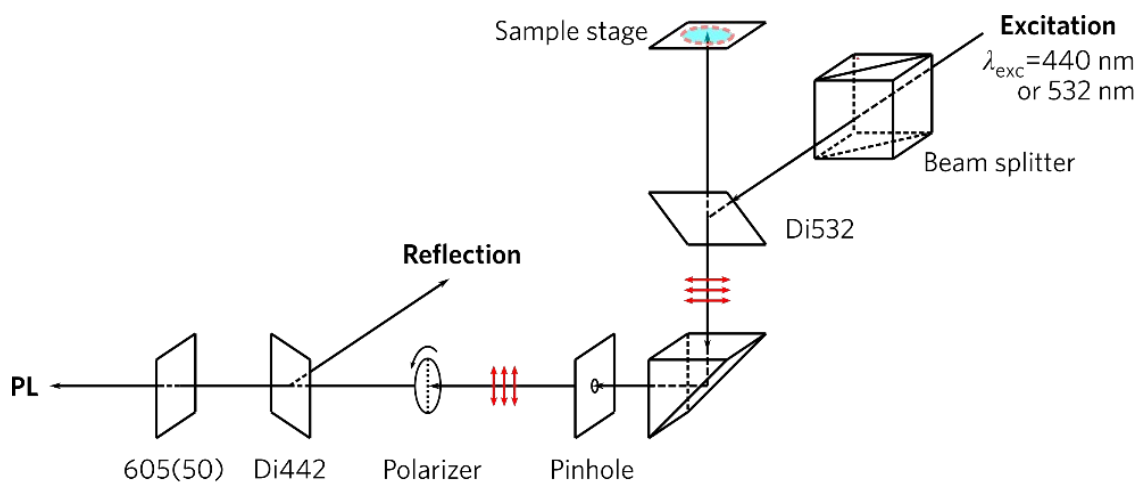

Figure S25. The instrumental setup for detection of emission polarization from individual light-emitting nanoclusters. By rotating the linear polarizer in front of the PL detector, the PL intensities as a function of the emission polarization angles are collected.

\subsection{Excitation polarization detection}

The sandwiched liquid samples for all the types of the nanoclusters were used to detect the excitation polarization effect. In the setup in Fig. S25, a halfwave plate is mounted upstream the sample. By rotating the waveplate by a speed of $10 \% \mathrm{~s}$, the excitation polarization directions were continuously changed (corresponding to a speed of $20 \% \mathrm{~s}$ ). The collected PL intensity profiles as a function of excitation polarization angle $\alpha$ were used to determine the excitation DOP and phases. 


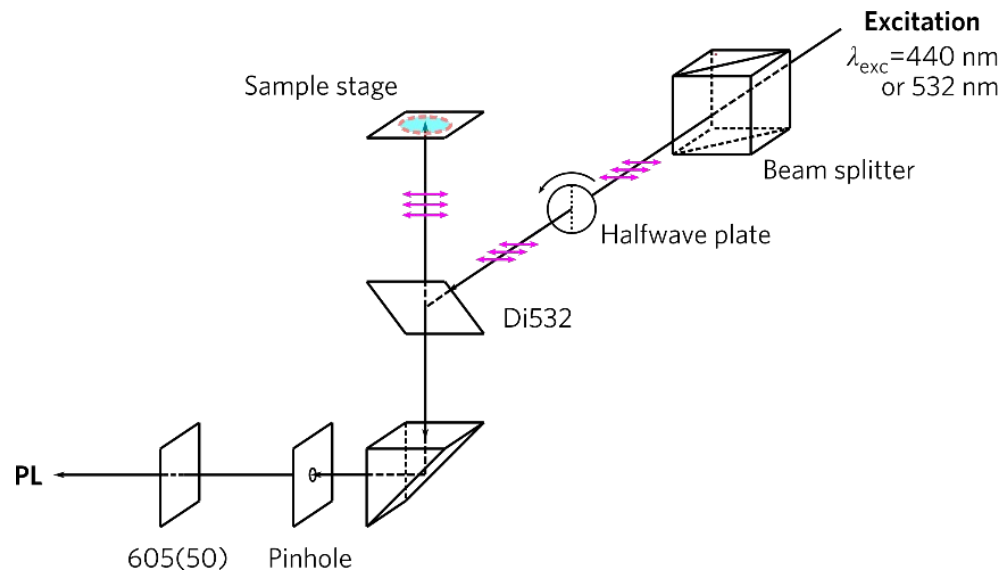

Figure S26. The instrumental setup for detection of excitation-polarization-dependent emission from individual light-emitting nanoclusters. By rotating the halfwave plate to continuously change excitation polarization directions, the PL intensities as a function of the excitation polarization angles $\alpha$ are collected.

During the detection of the emission polarization and the excitation polarization-dependent PL output, triggering the rotation of an analyzer or a half waveplate and collecting the PL intensity are controlled by two independent software, which are operated manually. The time shift between the initiation of the two acquisition software is about $1 \mathrm{~s}$, corresponding to $10^{\circ}$ error in $\theta$ phases and $20^{\circ}$ error in $\alpha$ phases. The software miss-synchronization between PL intensity acquisition software and waveplate or analyzer rotation software causes a slight mismatch of the phases for multiple measurements for a same cluster, such as in Fig. S7.

In order to check the potential polarization sensitivity of the optical detection setup, we used the concentrated QDs $\left(\lambda_{\text {emi }}=605 \mathrm{~nm}\right)$ dried on a coverslip as a reference. The concentrated QDs do not have preferred emission polarization directions and the PL intensities for all directions are approximately the same. We measured the reference by changing emission polarization directions (vertically and horizontally) and excitation polarization directions at both excitation wavelengths (440 nm and $532 \mathrm{~nm}$ ) using the setups in Fig. S25 and Fig. 26, respectively. Figs. S27a and S27b show vertically and horizontally polarized components at two excitation wavelengths, and Fig. S27c shows PL intensity profiles a function of excitation polarization angle $\alpha$. The $\mathrm{G}$ factor is defined as a ratio of the sensitivities of the detection system for vertically and horizontally polarized components $\left(G=S_{V} / S_{H}\right){ }^{2}$ As shown in Fig. S27, the $\mathrm{G}$ factor is approximately one $\left(\mathrm{G}_{440}=0.97\right.$ and $\left.\mathrm{G}_{532}=0.93\right)$. 

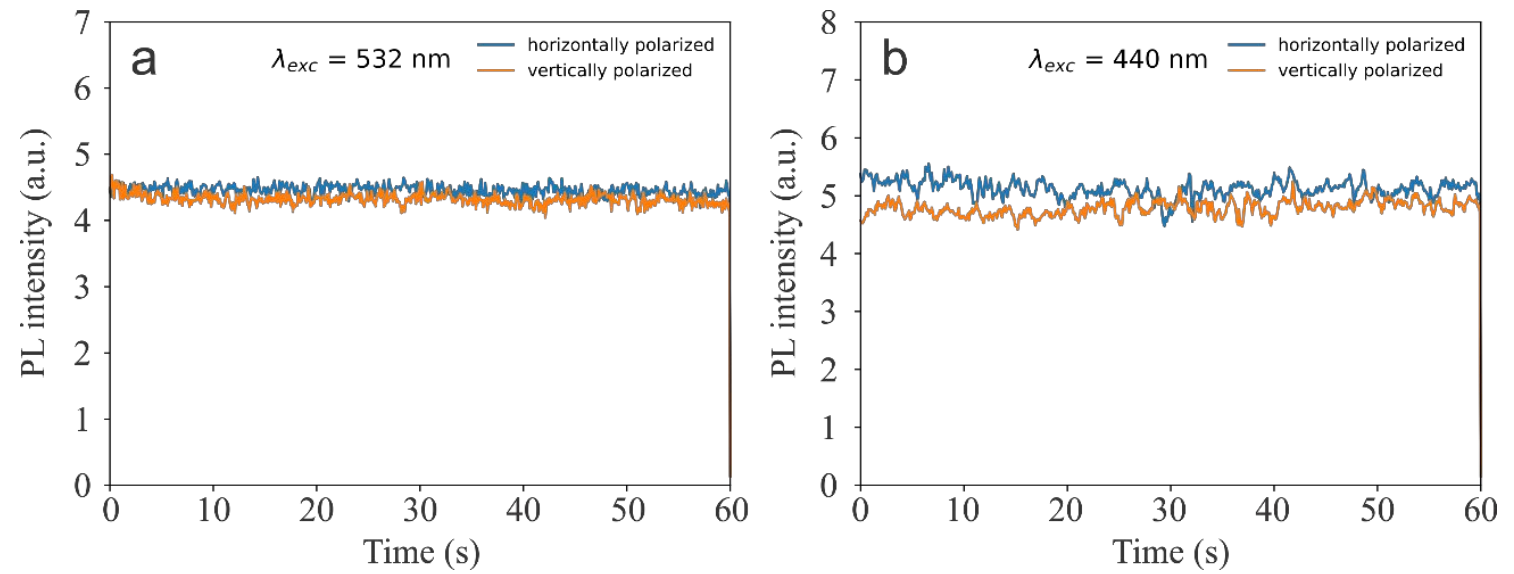

Figure S27. Polarization sensitivity of the detection system using concentrated QDs as a reference. The measured vertically and horizontally polarized components at two excitation wavelengths: (a) $532 \mathrm{~nm}$, (b) $440 \mathrm{~nm}$.

4.4 Extraction of polarization directions from intensity profiles

In order to determine the polarization directions, we first extracted phases from the measured intensity profiles by fitting using a sinusoidal function, which is expressed as

$$
I(\theta)=A \sin \left[2 \pi f\left(\theta+\varphi^{\prime}\right)\right]+D,
$$

where $A$ is the amplitude, $f$ is the frequency, $\varphi^{\prime}$ is the phase, and $D$ is the amplitude shift. The $\varphi^{\prime}$ is useful to correlate the polarization directions to the cluster structural orientations measured from the SEM images, but it is slightly different from the traditional phase defined in sine wave. For our clusters, $f=1 / \pi$ $\operatorname{rad}^{-1}, \varphi^{\prime}$ will be in the range of $[0, \pi]$ instead of $[0,2 \pi]$ for the traditional sine wave.

The polarization direction represents a direction where the intensity $I(\theta)$ turns to be maximum. The analyzer was initially located in the horizontal direction and rotated clockwise when the intensity profile was measured. Thus, we introduce an angle $\varphi_{\text {optical }}$ (related to the $\varphi^{\prime}$ ) to directly describe the polarization direction with respect the horizontal directions, and $\varphi_{\text {optical }}$ simply obeys that $2 \pi f\left(\theta+\varphi_{\text {optical }}\right)$ $=\pi / 2-2 \pi f\left(\theta+\varphi^{\prime}\right)$. When $f=1 / \pi \mathrm{rad}^{-1}, \varphi_{\text {optical }}=\pi / 4-\varphi^{\prime} . \varphi_{\text {optical }}$ will also be in the range of $[0, \pi]$.

\section{Theoretical considerations}

5.1 PL enhancement

The measured PL intensity is generally expressed as

$$
R_{P L}=\xi \phi \sigma I,
$$

where $l$ is the incident intensity, $\sigma$ is the absorption cross section, $\xi$ is the detection factor, and the quantum yield efficiency is $\phi=K_{\mathrm{r}} /\left(K_{\mathrm{r}}+K_{\mathrm{nr}}\right)=K_{\mathrm{r}} \tau$, with $\mathrm{K}_{\mathrm{r}}$ and $\mathrm{K}_{\mathrm{nr}}$ being radiative and nonradiative rate, respectively. Thus, $R_{P L}=\xi K_{r} \tau \sigma I$. 
The PL enhancement factor $\left(E F_{\mathrm{PL}}\right)$ between two different wavelengths on the same QD-AuNP nanocluster is expressed as ${ }^{3,4}$

$$
\mathrm{EF}_{\mathrm{PL}}=\frac{\mathrm{PL}(532 \mathrm{~nm}) / \operatorname{Abs}(532 \mathrm{~nm})}{\mathrm{PL}(440 \mathrm{~nm}) / \operatorname{Abs}(440 \mathrm{~nm})}
$$

where the $\mathrm{PL}\left(\lambda_{\text {exc }}\right)$ is the $\mathrm{PL}$ intensity measured at a specific excitation wavelength, and $A b s\left(\lambda_{\text {exc }}\right)$ is the corresponding absorbance of bare QDs without AuNPs. From Eq. 1, for nanoclusters at different excitation wavelengths, $\mathrm{K}_{\mathrm{r}(532)} / \mathrm{K}_{\mathrm{r}(440)}=\mathrm{R}_{\mathrm{PL}(532)} / \mathrm{R}_{\mathrm{PL}(440)} \times\left(\sigma_{(440)} / \sigma_{(532)}\right)=\mathrm{EF}_{\mathrm{PL}}$.

$\mathrm{EF}_{\mathrm{PL}}$ is calculated from the measured PL intensities and absorbance values (on versus off $\mathrm{SPR}$ ) to reveal the plasmonic-induced $\mathrm{PL}$ enhancement. Single-molecule $\mathrm{EF}_{\mathrm{PL}}$ for each type of the nanoclusters was measured and a mean $\mathrm{EF}_{\mathrm{PL}}$ for each nanocluster was calculated as the $\mathrm{EF}_{\mathrm{PL}}$ results roughly follow normal distributions (See the corresponding histogram plots in Fig. S15). Such PL enhancement factors were normalized to that of free QDs (Fig. S14) to be compared with the theoretical calculations,

\subsection{Energy transfer}

To understand the energy transfer from the QDs to the AuNPs, the energy transfer efficiency, defined as $E=1-\tau / \tau_{0}$ (with $\tau$ and $\tau_{0}$ being lifetime from nanoclusters and bare QDs, respectively) is analyzed using various theoretical models, including Forster resonance energy transfer (FRET) model and nanometal surface energy transfer (NSET) model as discussed below. All these models describe a distance-dependent energy transfer process and a general formula of energy transfer efficiency is given by ${ }^{5}$

$$
E=\frac{N}{N+\left({ }^{R} / R_{0}\right)^{n}}
$$

where $N$ is the number of the AuNPs (acceptors) in the proximity of a QD (donor), $R$ the AuNP-QD surfaceto-center distance, $R_{0}$ is the critical distance that corresponds to $E=50 \%$ for a single donor-acceptor pair, and the exponent $n$ is 6 for the FRET model while $n=4$ for the NSET models. ${ }^{5}$ In our case, $N=1$ (AuNP monomer) or 2 (AuNP dimer), $R$ is fixed to $32 \mathrm{~nm}$.

The lifetime for a series of the nanoclusters is extracted from the PL intensity decay. The observed dramatic lifetime quenching $\left(\tau / \tau_{0}<1\right)$ is the manifestation of the effect of energy transfer (Fig. S16). Such quenching effect also depends on the sizes and numbers of AuNPs. Specifically, the severe quenching arises from larger AuNPs; the AuNP dimer has stronger impact than the AuNP monomer. Among the tested nanoclusters, the most significant quenching was observed in the Oct-QD-Au50-2 consisting of two $50 \mathrm{~nm}$ AuNPs, and the resulting $\tau / \tau_{0}=0.35$. No clear lifetime difference was observed for optical excitation off and onto the SPR (between $440 \mathrm{~nm}$ and $532 \mathrm{~nm}$ ) of AuNPs.

Forster resonance energy transfer (FRET). FRET is a non-radiative transfer of energy resulting from longrange dipole-dipole interactions between a donor (D) molecule in excited state and an acceptor (A) molecule in the ground state. The energy transfer rate depends on optical characteristics of the donor and the acceptor and the distance between them. The critical distance $R_{\text {O(FRET) }}$ (also known as Forster distance) corresponds to the condition under which half the donors are deactivated by energy transfer and half deactivated by the usual radiative and non-radiative rates, i.e., the energy transfer efficiency $E=$ $50 \%$. The $R_{0(\text { FRET) }}$ is given by ${ }^{2}$ 


$$
R_{0(F R E T)}^{6}=\frac{9000(\ln 10) \kappa^{2} \emptyset_{D} J(\lambda)}{128 \pi^{5} N_{A} n_{m}^{4}}
$$

where $\emptyset_{D}$ is the quantum yield of the donor in the absence of the acceptor, $n_{\mathrm{m}}$ is the refractive index of the medium, $N_{\mathrm{A}}$ is the Avogadro's number, $K^{2}$ is an orientation factor depending on the relative orientation of the transition dipole of the donor and the acceptor, $J(\lambda)$ corresponds to the degree of spectral overlap between the donor emission and the acceptor absorption. The $J(\lambda)$ is expressed as ${ }^{2}$

$$
J(\lambda)=\frac{\int_{0}^{\infty} F_{D}(\lambda) \varepsilon_{e x t}(\lambda) \lambda^{4} d \lambda}{\int_{0}^{\infty} F_{D}(\lambda) d \lambda},
$$

where $F_{D}(\lambda)$ is the fluorescence spectrum of the donor at the wavelength $\lambda, \varepsilon_{\text {ext }}(\lambda)$ is the extinction coefficient spectrum of the acceptor at the wavelength $\lambda$. It is generally assumed that the orientation factor $\kappa^{2}$ is equal to $2 / 3$ for dynamic random averaging of the donor and the acceptor. ${ }^{2}$

In this study, the donor QDs have the characteristic emission wavelength $\lambda_{\mathrm{emi}}$ of $605 \mathrm{~nm}$, while the AuNP absorption peak positions (maximum SPR peak $\lambda_{\text {SPRmax }}$ ) are in the range of 525-535 nm (Fig. S28, S29). Overall, there is a poor overlap of the AuNP absorption spectra and the QD PL spectrum. However, as the AuNP sizes increase, the overlaps of AuNP extinction coefficient spectra and the QD PL spectrum rapidly increase (Fig. S28b), which leads to a size-dependent $J(\lambda)$ and Forster distance $R_{0 \text { (FRET). }}$

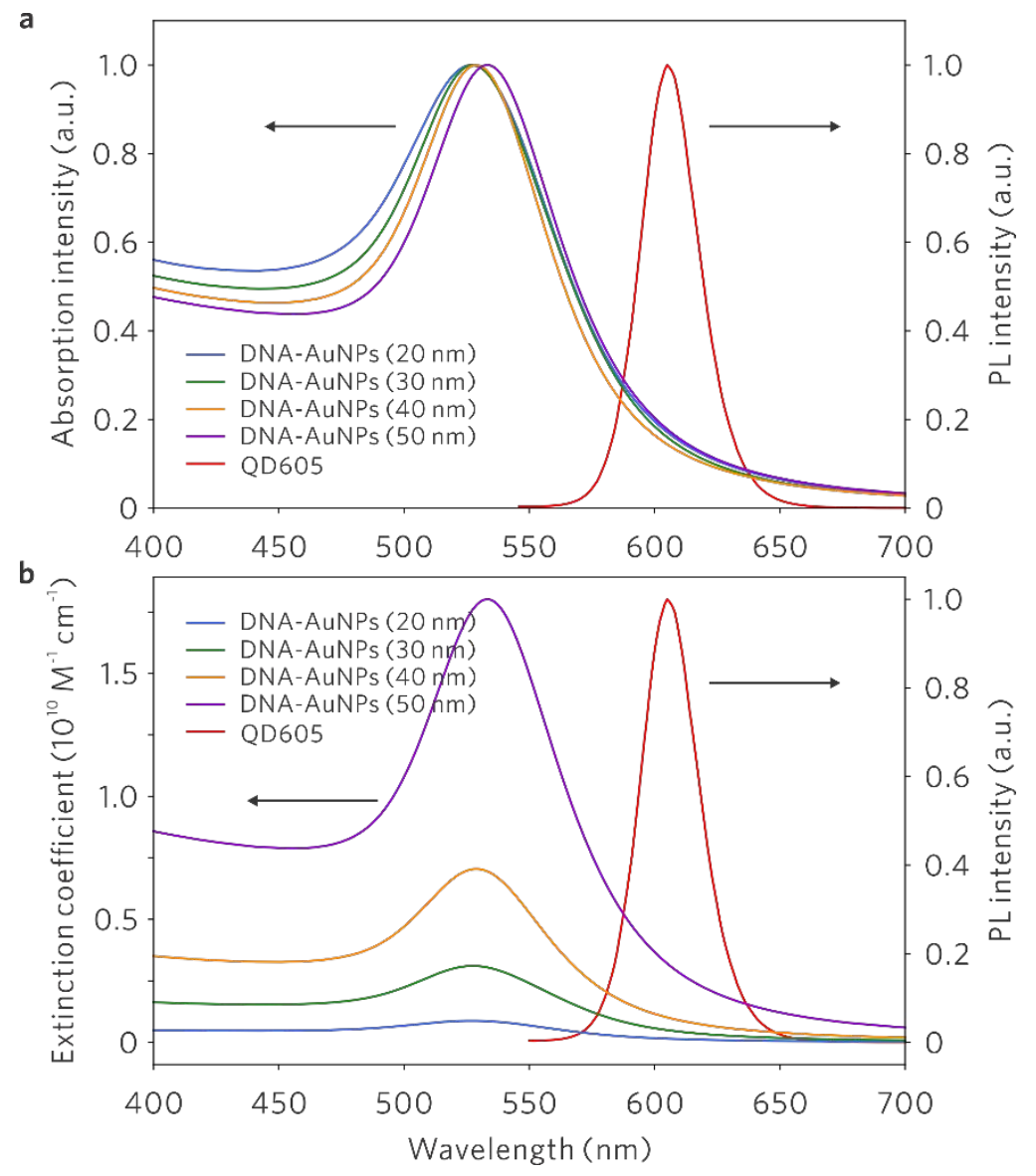


Figure S28. Spectra of the DNA-AuNP absorption and the QD PL. a, Overlap of the measured absorption spectra of DNA-AuNPs (20-50 nm) and the measured PL spectrum of the QDs. b, Overlap of the extinction coefficient spectra of AuNPs and the PL spectrum of QDs.

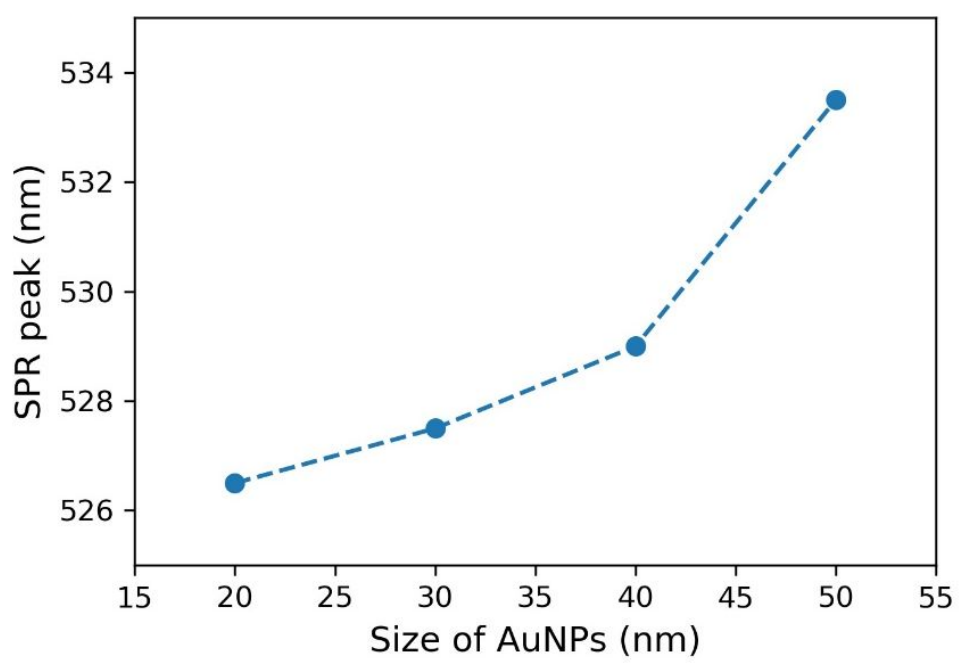

Figure S29. SPR peaks for AuNPs with various sizes $(20-50 \mathrm{~nm})$. The SPR shift to the larger wavelength when the size is increased.

Nanometal surface energy transfer (NSET). NSET describes a non-radiative energy transfer from a dipole to a noble metal surface. The characteristic length $R_{0 \text { (NSET) }}$ is given by ${ }^{5-7}$

$$
R_{0(N S E T)}^{4}=0.225 \frac{c n_{m}^{2} \emptyset_{D} \lambda_{e m i}^{2}}{4 \pi^{2} \omega_{F} k_{F}}
$$

where $c$ is the speed of light in vacuum $\left(c=299,792,458 \mathrm{~m} / \mathrm{s}\right.$ ), $\lambda_{\mathrm{emi}}$ is the donor emission wavelength, and $\omega_{\mathrm{F}}$ and $k_{\mathrm{F}}$ are Fermi frequency and Fermi wavevector for the metal, respectively. For bulk gold, $\omega_{\mathrm{F}}=8.4 \times$ $10^{15} \mathrm{~s}^{-1}$ and $k_{\mathrm{F}}=1.2 \times 10^{10} \mathrm{~m}^{-1} .5,6,8$

In the NSET model, the donor is considered as a point dipole and the surface of the metallic NP is assumed to be an infinite metal surface rather than a single dipole in the FRET model. Under such approximation, the size of the metallic NP is neglected, thus this model is size-independent.

Size-dependent NSET (sNSET). A modified NSET model to reflect the size-dependent effect is introduced by Breshike et. al. ${ }^{9}$. The size-dependent changes in dielectric constants and absorption cross sections of the metallic NPs are incorporated in the SNSET model, as shown in the characteristic length $R_{0 \text { (SNSET) }}$ given below $^{8-10}$ :

$$
R_{0(S N S E T)}^{4}=A_{e} \emptyset_{D} \frac{\alpha^{4} \lambda_{e m i}^{4} n_{r}}{n_{m}^{4} 2 n_{m}}\left(1+\frac{\varepsilon_{m}^{2}}{|\varepsilon|^{2}}\right)
$$

where $\varepsilon_{m}=n_{m}^{2}$ is the dielectric constant of the medium, $\varepsilon$ is the complex dielectric function of the metallic NPs $\left(\varepsilon=n^{2}=\varepsilon_{1}+i \varepsilon_{2}\right.$ and $\left.n=n_{r}+i k\right), \alpha$ is the geometric factor $\left(\alpha_{\|}=\sqrt[4]{9 / 2} /(4 \pi)\right.$ for a parallel dipole 
and $\alpha_{\perp}=\sqrt[4]{9} /(4 \pi)$ for a perpendicular dipole; for isotropic orientation, $\left.\alpha=1 / 3 \alpha_{\perp}+2 / 3 \alpha_{\|}\right)$, and $A_{\mathrm{e}}$ is the e-based NP absorbance. For a single NP, $A_{\mathrm{e}}$ can be expressed as

$$
A_{e}=\ln 10 \frac{\varepsilon_{e x t}(\lambda) d_{n p}}{10 N_{A} V_{n p}}
$$

where $d_{\mathrm{np}}$ is the NP diameter, $V_{\mathrm{np}}$ is the NP volume.

The complex dielectric functions consist of contributions from both bound electrons and free elections. When the size of NPs is comparable to or smaller than the mean free path of the conduction electrons in the bulk $\left(I_{\infty} \approx 42 \mathrm{~nm} \text { for gold }\right)^{11}$, collisions of the electrons with the particle surfaces leads to the sizedependence of the mean free path, which is given by ${ }^{11}$

$$
l(r)^{-1}=l_{\infty}^{-1}+r_{n p}^{-1},
$$

where $r$ is the radius of the NPs. By taking this effect into account and using reported complex dielectric functions of bulk materials ${ }^{12}$, the complex dielectric functions of AuNPs with different sizes are calculated $^{11,13}$ shown in Fig. S30.

a

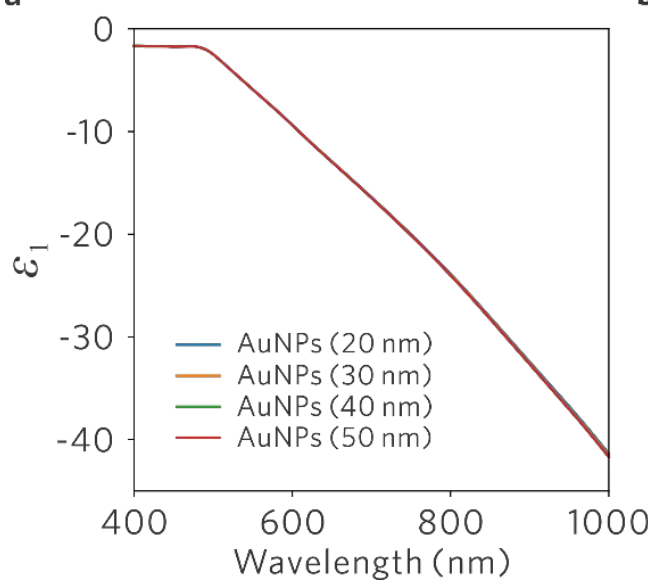

b

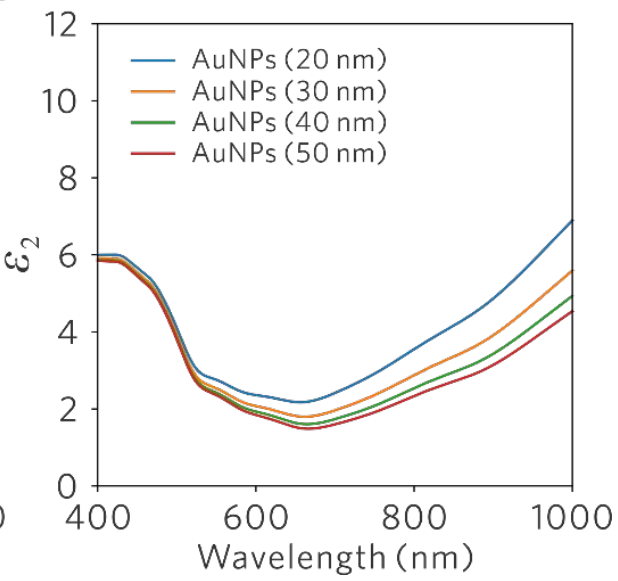

Figure S30. Calculated complex dielectric functions (real part $\varepsilon_{1}$ and imaginary part $\varepsilon_{2}$ ) of AuNPs (20-50 $\mathrm{nm}$ ) with mean free path correction. The experimental values of dielectric functions in the bulk materials are taken from Ref 12 and the calculation method is modified from Ref 13.

Based on the Mie theory ${ }^{11}$, the extinction cross sections $\sigma_{\text {ext }}$ and extinction coefficients $\varepsilon_{\text {ext }}$ for the AuNPs with various sizes are calculated using the complex dielectric functions above. The $\varepsilon_{\text {ext }}$ at the QD emission wavelength $\lambda_{\text {emi }}$ is used to calculate the size-dependent absorbance $A_{\mathrm{e}}$ and further $R_{0(\text { SNSET). }}$. 


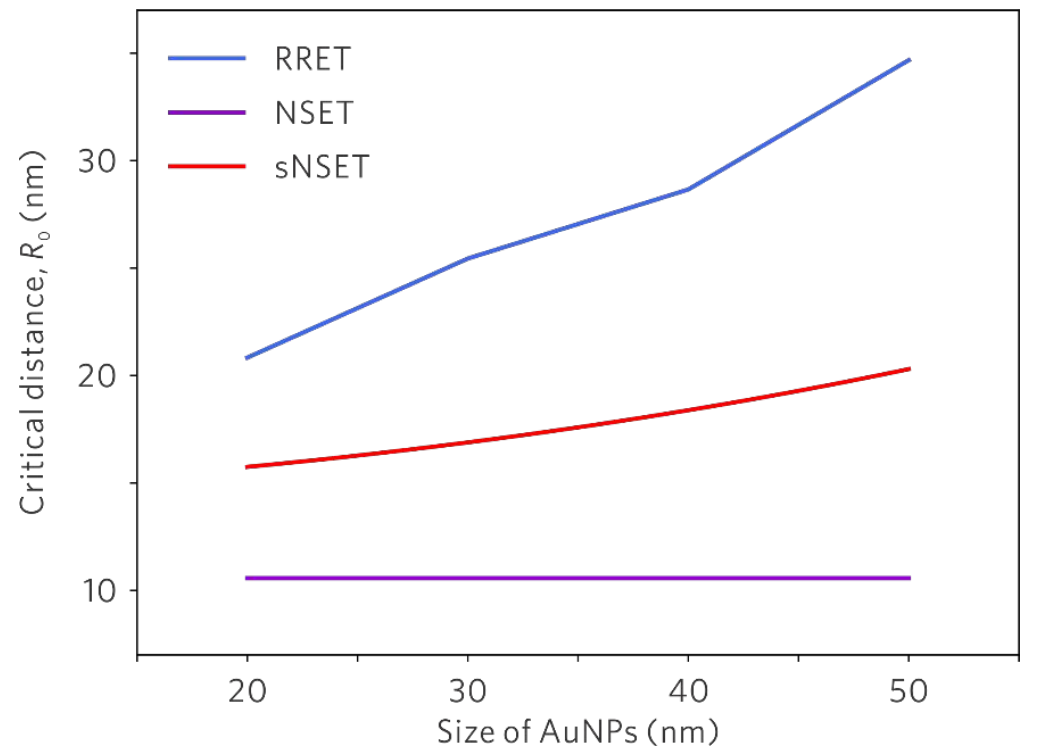

Figure S31. Critical energy transfer distance $R_{0}$ for the systems of the QDs $\left(\lambda_{\text {emi }}=605 \mathrm{~nm}\right)$ and the AuNPs with different sizes calculated based on three energy transfer models (FRET, NSET and SNSET).

By applying FRET, NSET and sNSET models to our nanocluster systems of the QDs $\left(\lambda_{\text {emi }}=605 \mathrm{~nm}\right)$ and the AuNPs with different sizes (20-50 nm), the critical distance $R_{0}$ for these models are calculated (Fig. 31). Here, we use $n_{\mathrm{m}}=1.76$ for the DNA-based medium between the QDs and the AuNPs, and $\emptyset_{D}=0.65$ as the specifications provided by the company state $\emptyset_{D} \geq 0.65$. As shown in Fig. S31, $R_{\text {O(NSET) }}=10.6 \mathrm{~nm}, R_{0 \text { (FRET) }}$ $=20.8-34.7 \mathrm{~nm}$, and $R_{\text {O(SNSET) }}=15.8-20.3 \mathrm{~nm}$. In the nanoclusters, the AuNP surface-to-QD center distance $R$ is around $32 \mathrm{~nm}$. The corresponding energy transfer efficiencies $E$ for the nanoclusters with the AuNP monomers and dimers are plotted in Fig. S17. Overall, the SNSET model shows a good prediction, while FRET and NSET models overestimate and underestimate the energy transfer, respectively.

\subsection{Simulation}

Lumerical FDTD. Full-field, 3D simulations were performed using Lumerical FDTD, a commercial electromagnetics software package. Perfectly matched layer (PML) boundary conditions were used on all edges of the simulation region to simulate infinite free space; symmetry boundary conditions were also applied to reduce computation time. The simulation region contained two gold nanoparticles (Palik refractive index data) spaced $64 \mathrm{~nm}$ apart (edge-to-edge) with equal diameters (20, 30, 40 or 50nm) and the background index was set to 1.4 to coincide with the index of the glass slide and the DNA layer. A single-wavelength (532 or $440 \mathrm{~nm}$ ) plane wave source was used for illumination, with polarization parallel and perpendicular to the gold nanoparticle dumbbell axis. The electric field intensity in the middle of the two gold nanoparticles was recorded and the enhancement factor was calculated as the ratio of the electric field intensity with the gold nanoparticles to the electric field intensity without the gold nanoparticles.

Comsol FEM. 3D finite element modeling were conducted using Comsol Multiphysics software. For emission polarization calculations, a dipole moment was placed in the position of the QD in the nanoclusters. The dipole vector directions were chosen to be parallel or perpendicular to the nanocluster axes. The radiation power was integrated from whole space. For excitation polarization calculation, only AuNP nanostructures were built and a Gaussian beam with corresponding wavelengths was focused on 
the AuNP structures. The electric field intensity around the AuNP structures were calculated. FEM simulations emission and excitation of monovalent clusters with $50 \mathrm{~nm}$ AuNPs are provided below.
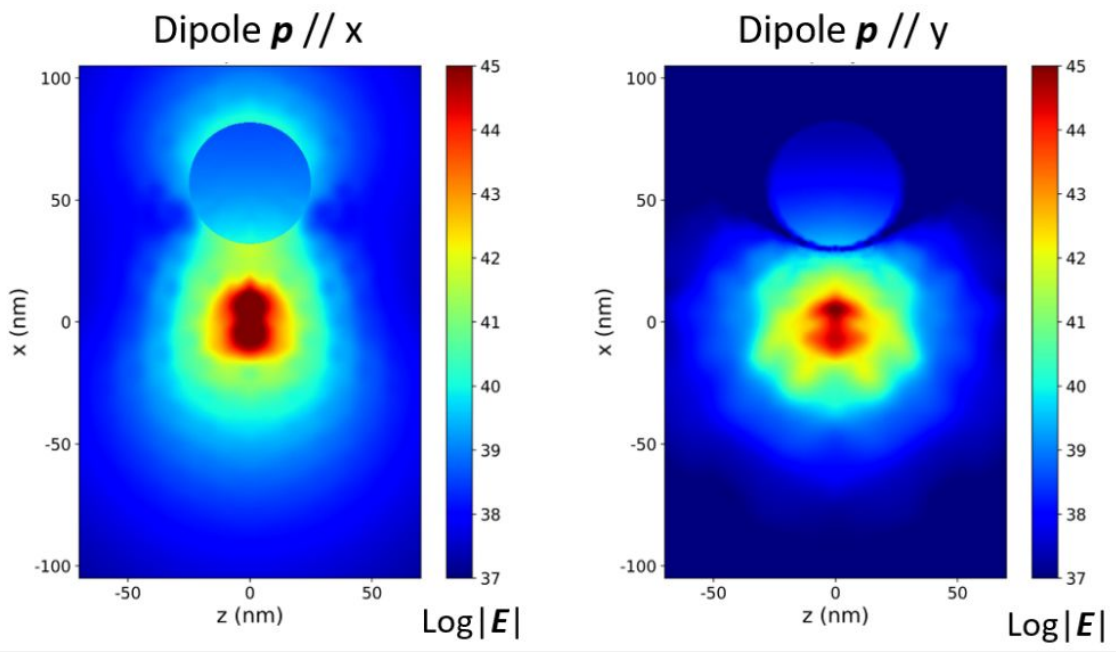

Figure S32. The relative electric field distribution based on the FEM simulation from a point dipole of $\lambda_{\text {emi }}$ $=605 \mathrm{~nm}$ in the position of QD for monovalent cluster Oct-QD-Au50-1 with the dipole vector parallel to $\mathrm{x}$ (cluster axis) or $\mathrm{y}$. The degree of polarization for radiation power is 0.46 .

a

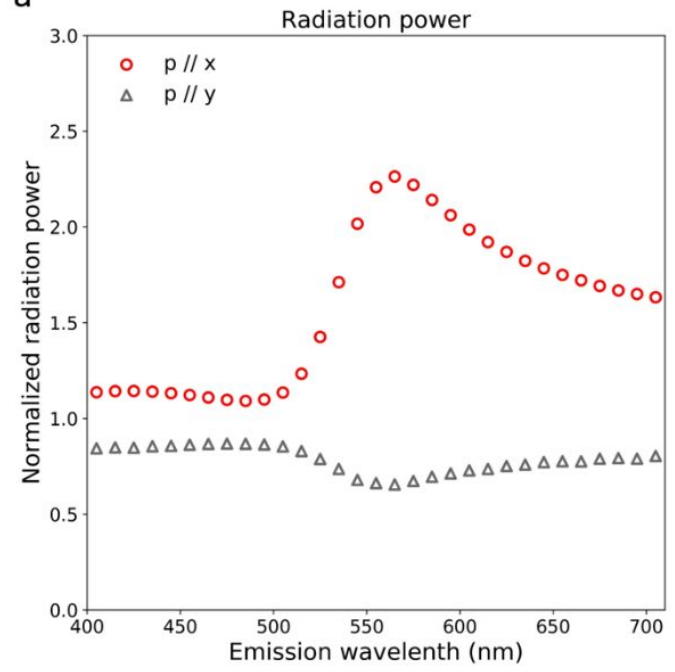

b

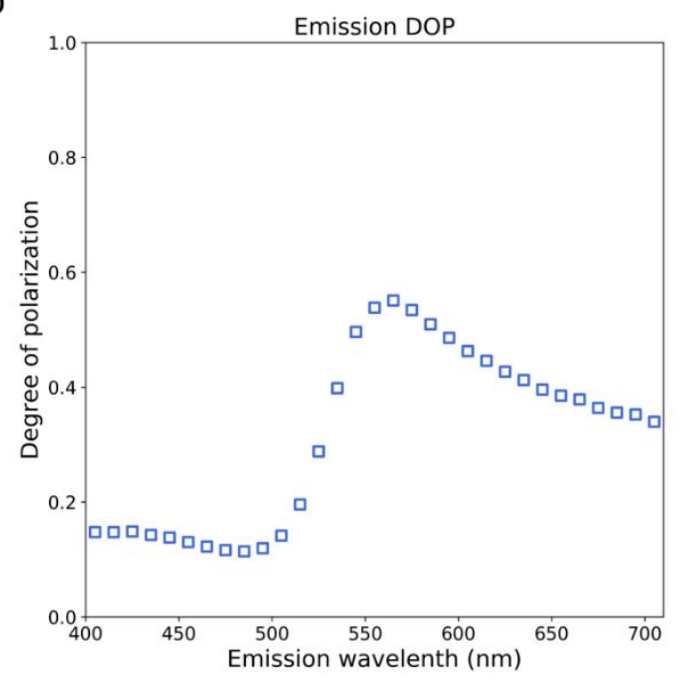

Figure S33. FEM-simulated emission polarization of a dipole moment in a monovalent nanocluster OctQD-Au50-1 with various levels of detuning between emission wavelengths and surface plasmon of AuNPs. $a$, Normalized radiation power of a point dipole moment with different emission wavelengths and with its vector parallel $(\boldsymbol{p} / / \mathrm{x})$ and perpendicular ( $\boldsymbol{p} / / \mathrm{y}$ ) to the nanocluster axis ( $\mathrm{x}$ axis). b, DOP of radiation power as function of the emission wavelengths. 


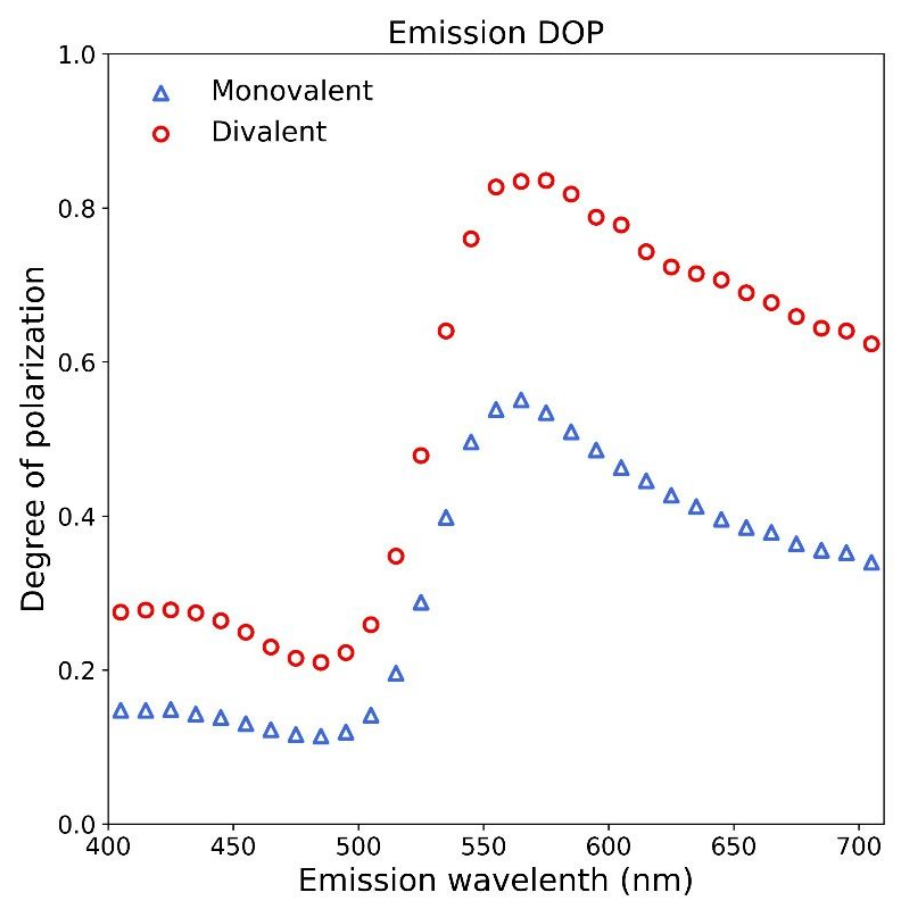

Figure S34. FEM-simulated DOP of radiation power of a dipole moment in a mono- and divalent nanoclusters of $50 \mathrm{~nm}$ AuNPs (Oct-QD-Au50-1 and Oct-QD-Au50-2) with various levels of detuning between emission wavelengths and surface plasmon of AuNPs. The increase of valence strongly enhances the emission polarization for each emission wavelength. 

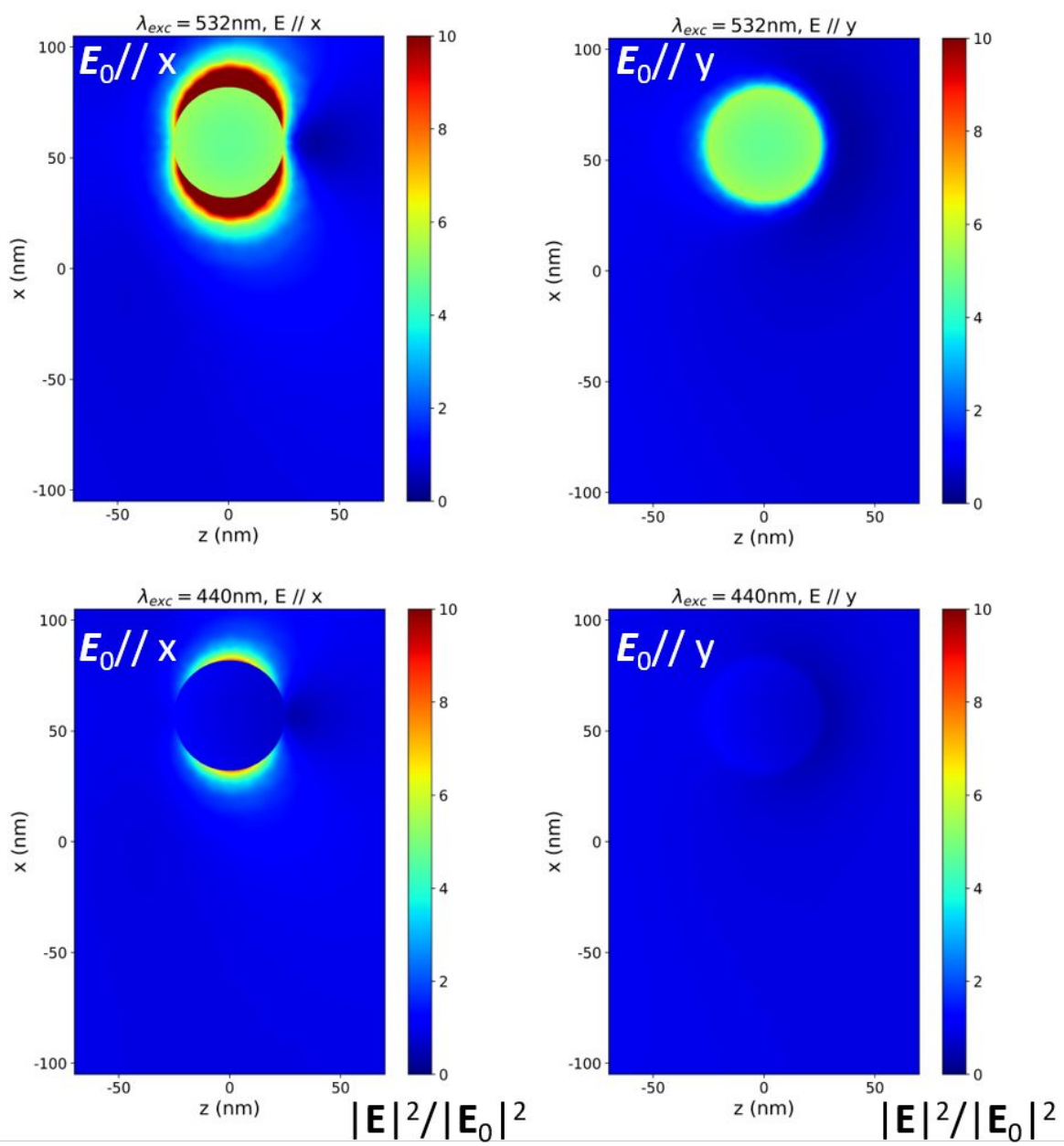

Figure S35. FEM-simulated electric field enhancement $|E|^{2} /\left|E_{0}\right|^{2}$ for a monovalent cluster with the same structural parameters to the nanocluster Oct-QD-Au50-1 at $\lambda_{\text {exc }}=532 \mathrm{~nm}$ (a) and $440 \mathrm{~nm}$ (b) based on the Comsol simulation. The propagation of the field is along the $z$ axis and the polarization is along the $x$ and $y$ axes, respectively. 

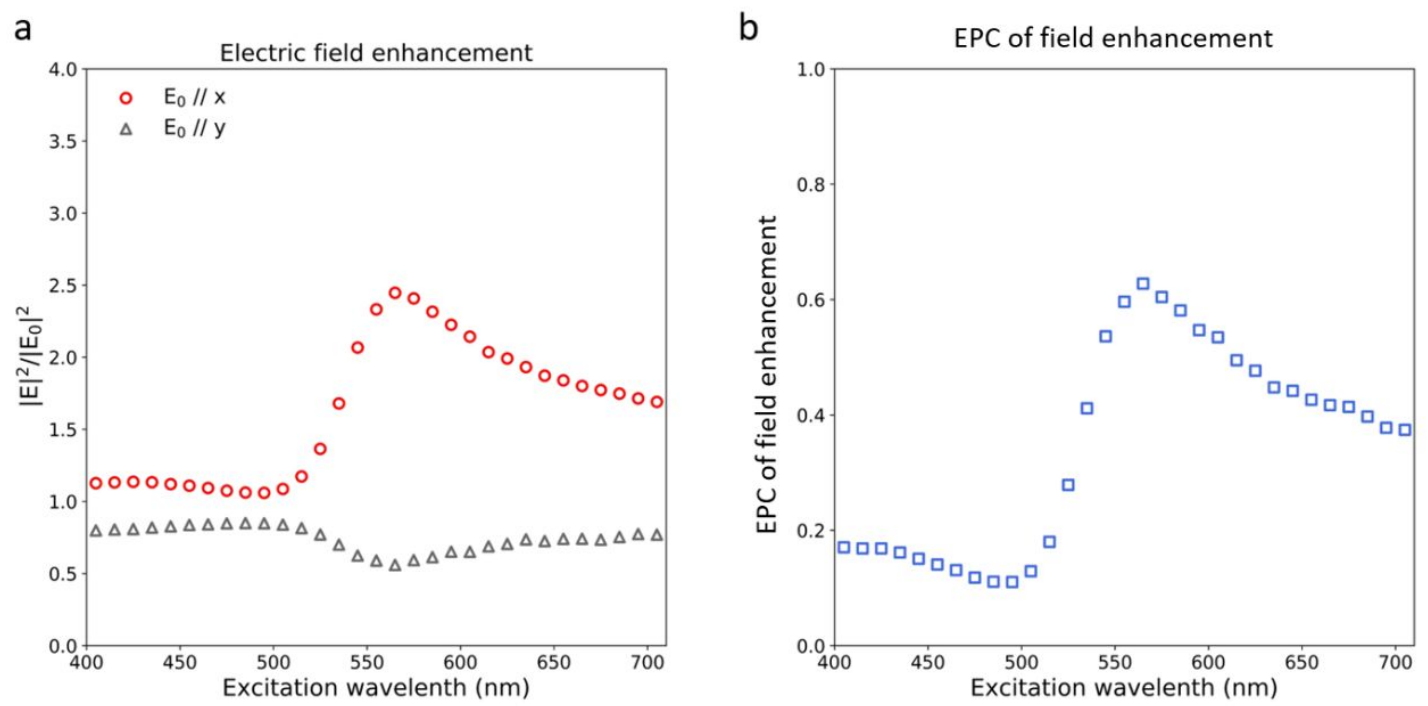

Figure S36. FEM-simulated electric field enhancement and EPC of field enhancement of a monovalent nanocluster with AuNP size of $50 \mathrm{~nm}$ (Oct-QD-Au50-1) with various excitation photon energies. a, Electric field enhancement in the position of the QD for excitation polarization direction parallel $\left(E_{0} / / x\right)$ and perpendicular $\left(E_{0} / / y\right)$ to the nanocluster axis (x axis). b, EPC of field enhancement as a function of the excitation wavelengths. Excitation around SPR increases the EPC of the field enhancement. 


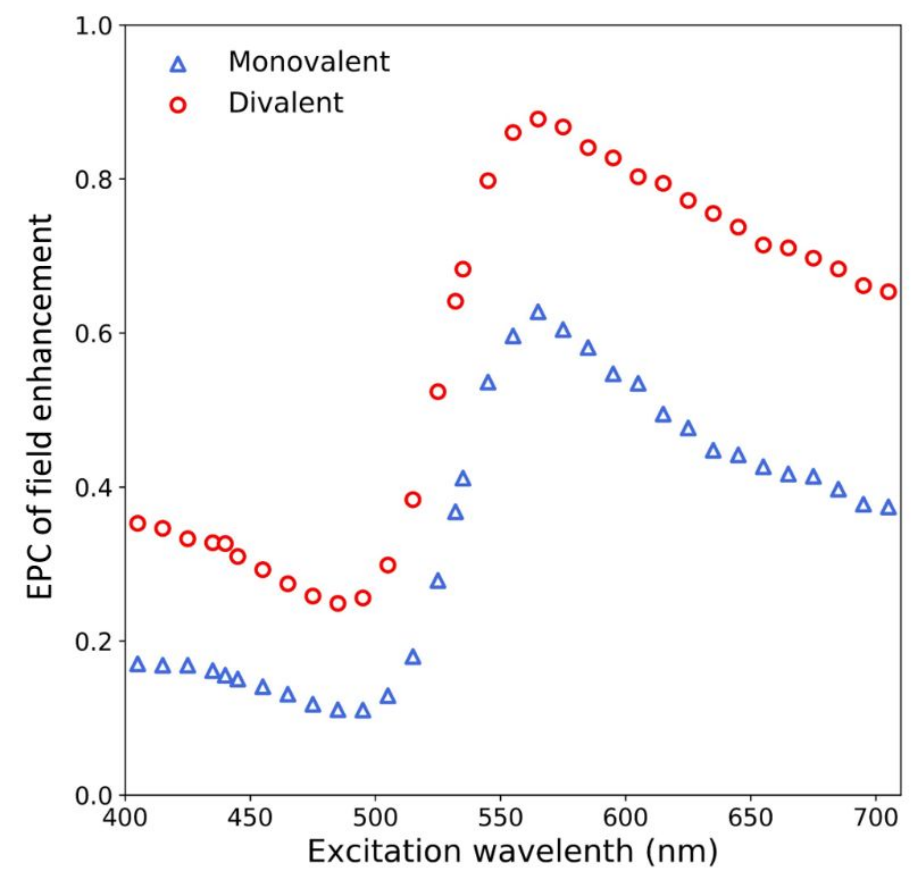

Figure S37. FEM-simulated EPC of field enhancement in a mono- and divalent nanoclusters of $50 \mathrm{~nm}$ AuNPs (Oct-QD-Au50-1 and Oct-QD-Au50-2) with various levels of excitation photon energies. The increase of valence strongly enhances the EPC for each excitation wavelength. Excitation around SPR increases the EPC of the field enhancement.

\section{DNA sequences}

6.1 Origami staples (112 strands)

$\begin{array}{ll}\text { Name } & \text { Sequence (5'-3') } \\ \text { Bundle1_1 } & \text { GCCTTGAATCTTTTCCGGAACCGCCTCCCAGAGCCCAGAGCCGCCGCCAGCATT } \\ \text { Bundle1_2 } & \text { ATAAGGCGCCAAAAGTTGAGATTTAGGATAACGGACCAGTCA } \\ \text { Bundle1_4 } & \text { GATGGTTGGGAAGAAAAATCCACCAGAAATAATTGGGCTTGA } \\ \text { Bundle1_5 } & \text { GGACGTTTAATTTCGACGAGAAACACCACCACTAATGCAGAT } \\ \text { Bundle1_6 } & \text { ATTTTAAGAACTGGCTTGAATTATCAGTGA } \\ \text { Bundle1_7 } & \text { TCAGAACCGCCACCCTCTCAGAGTATTAGC } \\ \text { Bundle1_8 } & \text { TCAGAGCGCCACCACATAATCAAAATCAGAACGAGTAGTATG } \\ \text { Bundle1_9 } & \text { AACCAGACGCTACGTTAATAAAACGAACATACCACATTCAGG } \\ \text { Bundle1_11 } & \text { GTTTGCCTATTCACAGGCAGGTCAGACGCCACCACACCACCC } \\ \text { Bundle1_12 } & \text { ACATAACTTGCCCTAACTTTAATCATTGCATTATAACAACATTATTACAGGTAG } \\ \text { Bundle2_2 } & \text { CATGTCACAAACGGCATTAAATGTGAGCAATTCGCGTTAAAT } \\ \text { Bundle2_4 } & \text { AAGATTGTTTTTAACCAAGAAACCATCGACCCAAAAACAGG } \\ \text { Bundle2_5 } & \text { CAGCTCATATAAGCGTACCCCGGTTGATGTGTCGGATTCTCC }\end{array}$




\begin{tabular}{|c|c|}
\hline Bundle2_6 & GTTAAAATTCGCATTATAAACGTAAACTAG \\
\hline Bundle2_7 & AGCACCATTACCATTACAGCAAATGACGGA \\
\hline Bundle2_8 & GTCACCAGAGCCATGGTGAATTATCACCAATCAGAAAAGCCT \\
\hline Bundle2_9 & CCGACTTATTAGGAACGCCATCAAAAATGAGTAACAACCCCA \\
\hline Bundle2_11 & AATTATTGTTTTCATGCCTTTAGCGTCAGATAGCACGGAAAC \\
\hline Bundle3_1 & CTTCGCTGGGCGCAGACGACAGTATCGGGGCACCGTCGCCATTCAGGCTGCGCA \\
\hline Bundle3_2 & ACAAAGAAATTTAGGTAGGGCTTAATTGTATACAACGGAATC \\
\hline Bundle3_4 & TGACCTACTAGAAAAAGCCCCAGGCAAAGCAATTTCATCTTC \\
\hline Bundle3_5 & ATAATTAAATTTAAAAAACTTTTTCAAACTTTTAAACAACGCC \\
\hline Bundle3_6 & AGGCGTTAAATAAGAAGACCGTGTCGCAAG \\
\hline Bundle3_7 & TGCCGGAAGGGGACTCGTAACCGTGCATTATATTTTAGTTCT \\
\hline Bundle3_8 & CTCCAGCCAGCTTTCCCCTCAGGACGTTGG \\
\hline Bundle3_9 & CAGTTTGAATGTTTAGTATCATATGCGTAGAATCGCCATAGC \\
\hline Bundle3_11 & TGTAGATATTACGCGGCGATCGGTGCGGGCGCCATCTTCTGG \\
\hline Bundle3_12 & AACATGTACGCGAGTGGTTTGAAATACCTAAACACATTCTTACCAGTATAAAGC \\
\hline Bundle4_1 & CGCTGGTGCTTTCCTGAATCGGCCAACGAGGGTGGTGATTGCCCTTCACCGCCT \\
\hline Bundle4_2 & AACAAAAATAACTAGGTCTGAGAGACTACGCTGAGTTTCCCT \\
\hline Bundle4_4 & AACAGTACTTGAAAACATATGAGACGGGTCTTTTTTAATGGA \\
\hline Bundle4_5 & TAGAATCCATAAATCATTTAACAATTTCTCCCGGCTTAGGTT \\
\hline Bundle4_6 & GTAAATCGTCGCTATTGAATAACTCAAGAA \\
\hline Bundle4_7 & TTGCGTATTGGGCGCCCGCGGGGTGCGCTC \\
\hline Bundle4_8 & TTTCACCGCATTAAAGTCGGGAAACCTGATTTGAATTACCCA \\
\hline Bundle4_9 & GCCAGCTAGGCGATAGCTTAGATTAAGACCTTTTTAAACCTGT \\
\hline Bundle4_11 & ACTGCCCTTGCCCCGTTGCAGCAAGCGGCAACAGCTTTTTTCT \\
\hline Bundle4_12 & GGGTTATTTAATTACAATATATGTGAGTAATTAATAAGAGTCAATAGTGAATTT \\
\hline Bundle5_1 & TCCAAATCTTCTGAATTATTTGCACGTAGGTTTAACGCTAACGAGCGTCTTTTCC \\
\hline Bundle5_2 & CAGATATTACCTGAATACCAAGTTACAATCGGGAGCTATTTT \\
\hline Bundle5_4 & ACGCGAGGCTACAACAGTACCTTTTACAAATCGCGCAGAGAA \\
\hline Bundle5_5 & GCACCCAGCGTTTTTTATCCGGTATTCTAGGCGAATTATTCA \\
\hline Bundle5_6 & AAGCCTTAAATCAAGACTTGGGGAGCAAAT \\
\hline Bundle5_7 & ATTGCGTAGATTTTCAAAACAGATTGTTTG \\
\hline Bundle5_8 & TGAATATTATCAAAATAATGGAAGGGTTAATATTTATCCCAA \\
\hline Bundle5_9 & CCTACCAACAGTAATTTTATCCTGAATCAAACAGCCATATGA \\
\hline Bundle5_11 & GATTATAAAGAAACGCCAGTTACAAAATTTACCAACGTCAGA \\
\hline Bundle5_12 & TTTCAATAGAAGGCAGCGAACCTCCCGATTAGTTGAAACAATAACGGATTCGCC \\
\hline Bundle6_1 & GATATTCTAAATTGAGCCGGAACGAGGCCCAACTTGGCGCATAGGCTGGCTGAC \\
\hline Bundle6_2 & GGTTGATTTTCCAGCAGACAGCCCTCATTCGTCACGGGATAG \\
\hline Bundle6_4 & AGTACCGAATAGGAACCCAAACGGTGTAACCTCAGGAGGTTT \\
\hline Bundle6_5 & CAAGCCCCCACCCTTAGCCCGGAATAGGACGATCTAAAGTTT \\
\hline Bundle6_6 & CAGAGCCACCACCCTCTCAGAACTCGAGAG \\
\hline Bundle6_7 & AAGGGAACCGAACTGAGCAGACGGTATCAT \\
\hline Bundle6_8 & GGACAGAGTTACTTTGTCGAAATCCGCGTGTATCACCGTACG \\
\hline Bundle6_9 & GCTCCATTGTGTACCGTAACACTGAGTTAGTTAGCGTAACCT \\
\hline Bundle6_11 & CGCCTGAATTACCCTAATCTTGACAAGACAGACCATGAAAGA \\
\hline
\end{tabular}




\begin{tabular}{|c|c|}
\hline Bundle6_12 & TGTCGTCATAAGTACAGAACCGCCACCCATTTTCACAGTACAAACTACAACGCC \\
\hline Bundle7_1 & ATGACCACTCGTTTGGCTTTTTGCAAAAGTTAGACTATATTCATTGAATCCCCCT \\
\hline Bundle7_2 & GTAATACGCAAACATGAGAGATCTACAACTAGCTGAGGCCGG \\
\hline Bundle7_4 & AGAACCCCAAATCACCATCTGCGGAATCGAATAAAAATTTTT \\
\hline Bundle7_5 & AGACAGTTCATATAGGAGAAGCCTTTATAACATTGCCTGAGA \\
\hline Bundle7_6 & GTAAAGATTCAAAAGGCCTGAGTTGACCCT \\
\hline Bundle7_7 & GGTAATAGTAAAATGTAAGTTTTACACTAT \\
\hline Bundle7_8 & GTCCAATAGCGAGAACCAGACGACGATATTCAACGCAAGGGA \\
\hline Bundle7_9 & CCAAAATACAATATGATATTCAACCGTTAGGCTATCAGGTAA \\
\hline Bundle7_11 & CATAACCTAAATCAACAGTTCAGAAAACGTCATAAGGATAGC \\
\hline Bundle7_12 & GTCTGGATTTTGCGTTTTAAATGCAATGGTGAGAAATAAATTAATGCCGGAGAG \\
\hline Bundle8_1 & GGGCGACCCCAAAAGTATGTTAGCAAACTAAAAGAGTCACAATCAATAGAAAAT \\
\hline Bundle8_2 & TATAAAGCATCGTAACCAAGTACCGCACCGGCTGTAATATCC \\
\hline Bundle8_4 & CAACATGATTTACGAGCATGGAATAAGTAAGACGACAATAAA \\
\hline Bundle8_5 & CATCCTATTCAGCTAAAAGGTAAAGTAAAAAGCAAGCCGTTT \\
\hline Bundle8_6 & GATAAGTCCTGAACAACTGTTTAAAGAGAA \\
\hline Bundle8_7 & TAAAGGTGGCAACATAGTAGAAAATAATAA \\
\hline Bundle8_8 & AGACACCTTACGCAGAACTGGCATGATTTTCTGTCCAGACAA \\
\hline Bundle8_9 & CTCCTTAACGTAGAAACCAATCAATAATTCATCGAGAACAGA \\
\hline Bundle8_11 & CGGAATAATTCAACCCAGCGCCAAAGACTTATTTTAACGCAA \\
\hline Bundle8_12 & TTATTTTTACCGACAATGCAGAACGCGCGAAAAATCTTTTCCTTATCATTCCAAG \\
\hline Bundle9_2 & CAGCCTTGGTTTTGTATTAAGAGGCTGACTGCCTATATCAGA \\
\hline Bundle9_4 & GGAAGCGCCCACAAACAGTTAATGCCCCGACTCCTCAAGATA \\
\hline Bundle9_5 & GAGATAACATTAGAAGAATAACATAAAAAGGAAGGATTAGGA \\
\hline Bundle9_6 & GTCAGAGGGTAATTGAGAACACCAAAATAG \\
\hline Bundle9_7 & AAGTTTTAACGGGGTCGGAGTGTAGAATGG \\
\hline Bundle9_8 & CAGTGCCTACATGGGAATTTACCGTTCCACAAGTAAGCAGAT \\
\hline Bundle9_9 & AGCGTCACGTATAAGAATTGAGTTAAGCCCTTTTTAAGAAAG \\
\hline Bundle9_11 & AAAGCGCCAAAGTTTATCTTACCGAAGCCCAATAATGAGTAA \\
\hline Bundle10_2 & TGCTAAACAGATGAAGAAACCACCAGAATTTAAAAAAAGGCT \\
\hline Bundle10_4 & GAGAATAGAGCCTTACCGTCTATCAAATGGAGCGGAATTAGA \\
\hline Bundle10_5 & CCAAAAGGAAAGGACAACAGTTTCAGCGAATCATCATATTCC \\
\hline Bundle10_6 & TTCACGTTGAAAATCTTGCGAATGGGATTT \\
\hline Bundle10_7 & GTCCACTATTAAAGAACCAGTTTTTGGTTCC \\
\hline Bundle10_8 & TCAAAGGGAGATAGCCCTTATAAATCAAGACAACAACCATCG \\
\hline Bundle10_9 & ATAGCCCGCGAAAATAATTGTATCGGTTCGCCGACAATGAGT \\
\hline Bundle10_11 & GAAATCGATAACCGGATACCGATAGTTGTATCAGCTCCAACG \\
\hline Bundle11_2 & ATTAAGTATAAAGCGGCAAGGCAAAGAAACTAATAGGGTACC \\
\hline Bundle11_4 & CACGACGAATTCGTGTGGCATCAATTCTTTAGCAAAATTACG \\
\hline Bundle11_5 & CAGGTCGACTCTAGAGCAAGCTTCAAGGCG \\
\hline Bundle11_6 & TAACCTGTTTAGCTATTTTTCGCATTCATTC \\
\hline Bundle11_7 & GAGCTCGTTGTAAACGCCAGGGTTTTCCAAAGCAATAAAGCC \\
\hline Bundle11_8 & CGCGAGCTTAGTTTTTCCCAATTCTGCGCAAGTGTAAAGCCT \\
\hline Bundle11_9 & AGTAGATTGAAAAGAATCATGGTCATAGCCGGAAGCATAAGT \\
\hline
\end{tabular}




$\begin{array}{ll}\text { Bundle11_11 } & \text { CATATAACTAATGAACACAACATACGAGCTGTTTCTTTGGGG } \\ \text { Bundle12_1 } & \text { ATGTTTTGCTTTTGATCGGAACGAGGGTACTTTTTCTTTTGATAAGAGGTCATT } \\ \text { Bundle12_2 } & \text { AGAAGCAACCAAGCCAAAAGAATACACTAATGCCAAAACTCC } \\ \text { Bundle12_4 } & \text { GAGGAAGCAGGATTCGGGTAAAATACGTAAAACACCCCCAG } \\ \text { Bundle12_5 } & \text { AACAGGTCCCGAATTGCATCAAAAAGATCTTTGATCATCAG } \\ \text { Bundle12_6 } & \text { TCAAAGCGAACCAGACCGTTTTATATAGTC } \\ \text { Bundle12_7 } & \text { GCTTTGAGGACTAAAGAGCAACGGGGAGTT } \\ \text { Bundle12_8 } & \text { AAGTTTCAGACAGCCGGGATCGTCACCCTTCTGTAGCTCAAC } \\ \text { Bundle12_9 } & \text { CAGCGAACATTAAAAGAGAGTACCTTTACTGAATATAATGAA } \\ \text { Bundle12_11 } & \text { AAAGGCCAAATATGTTAGAGCTTAATTGATTGCTCCATGAGG } \\ \text { Bundle12_12 } & \text { CGATTATAAGCGGAGACTTCAAATATCGCGGAAGCCTACGAAGGCACCAACCTA }\end{array}$

6.2 Inner anchoring staple (8 strands)

\begin{tabular}{|c|c|}
\hline Name & Sequence (5'-3') \\
\hline \multirow[t]{2}{*}{ Bundle2_1 } & ATCCATCACTTCATACTCTACGTTGTTGTTGTTGTTGTTGTAGCGCCATTAAATTGGGAATT \\
\hline & AGAGCGCAAGGCGCACCGTAATCAGTAGCGA \\
\hline \multirow[t]{2}{*}{ Bundle2_12 } & ATCCATCACTTCATACTCTACGTTGTTGTTGTTGTTGTTGTGGGAAATCATATAAATATTTAA \\
\hline & ATTGAATTTTTGTCTGGCCTTCCTGTAGCC \\
\hline Bundle9_1 & $\begin{array}{l}\text { ATCCATCACTTCATACTCTACGTTGTTGTTGTTGTTGTTAGCCGAAAGTCTCTCTTTTGATGA } \\
\text { TACAAGTGCCTTAAGAGCAAGAAACAATGA }\end{array}$ \\
\hline \multirow[t]{2}{*}{ Bundle9_12 } & ATCCATCACTTCATACTCTACGTTGTTGTTGTTGTTGTTTTAGCGGTACAGAGCGGGAGAAT \\
\hline & TAACTGCGCTAATTTCGGAACCTATTATTCT \\
\hline \multirow[t]{2}{*}{ Bundle10_1 } & ATCCATCACTTCATACTCTACGTTGTTGTTGTTGTTGTTCCCACGCGCAAAATGGTTGAGTG \\
\hline & TTGTTCGTGGACTTGCTTTCGAGGTGAATTT \\
\hline \multirow[t]{2}{*}{ Bundle10_12 } & ATCCATCACTTCATACTCTACGTTGTTGTTGTTGTTGTTTGATTATCAACTTTACAACTAAAG \\
\hline & GAATCCAAAAAGTTTGAGTAACATTATCAT \\
\hline \multirow[t]{2}{*}{ Bundle11_1 } & ATCCATCACTTCATACTCTACGTTGTTGTTGTTGTTGTTGGGGTGCCAGTTGAGACCATTAG \\
\hline & ATACAATTTTCACTGTGTGAAATTGTTATCC \\
\hline Bundle11_12 & $\begin{array}{l}\text { ATCCATCACTTCATACTCTACGTTGTTGTTGTTGTTGTTTCAGAGCTGGGTAAACGACGGCC } \\
\text { AGTGCGATCCCCGTAGTAGCATTAACATCCA }\end{array}$ \\
\hline
\end{tabular}

6.3 Outer anchoring staple (24 strands)

Monovalent cluster:

\author{
Name \\ Bundle5_A \\ Bundle6_A \\ Bundle9_A \\ Bundle10_A \\ Bundle1_B \\ Bundle6_B \\ Bundle7_B \\ Bundle12_B \\ Bundle2_C
}

\section{Sequence (5'-3')}

AGAGCCTAATTTGATTTTTTGTTTAAATCCTGAAATAAAGAA

TGTAGCATTCCAACGTTAGTAAATGAAGTGCCGCGCCACCCT

GAAACATGAAAGCTCAGTACCAGGCGAAAAATGCTGAACAAA

TTTGCGGAACAATGGCAATTCATCAATCTGTATAATAATTTT

AAAGATTCATCAGGAATTACGAGGCATGCTCATCCTTATGCGAAAAAAAAAAAAAAA CTTCATCAAGAGAAATCAACGTAACAGAGATTTGTCAATCATAAAAAAAAAAAAAAA CAAATGCTTTAAAAAATCAGGTCTTTAAGAGCAGCCAGAGGGAAAAAAAAAAAAAAA AAACGAAAGAGGGCGAAACAAAGTACTGACTATATTCGAGCTAAAAAAAAAAAAAAA AGCTTTCATCAACGGATTGACCGTAAAATCGTATAATATTTT 


$\begin{array}{ll}\text { Bundle3_C } & \text { ACTGTTGGGAAGCAGCTGGCGAAAGGATAGGTCAAGATCGCA } \\ \text { Bundle7_C } & \text { GGTAGCTATTTTAGAGAATCGATGAAAACATTAAATGTGTAG } \\ \text { Bundle11_C } & \text { ATAAATCATACATAAATCGGTTGTACTGTGCTGGCATGCCTG } \\ \text { Bundle3_D } & \text { CAACGCTCAACAGCAGAGGCATTTTCAATCCAATGATAAATA } \\ \text { Bundle4_D } & \text { ATCAAAATCATATATGTAAATGCTGAACAAACACTTGCTTCT } \\ \text { Bundle5_D } & \text { TGATTGCTTTGAGCAAAAGAAGATGAAATAGCAGAGGTTTTG } \\ \text { Bundle8_D } & \text { AACGGGTATTAAGGAATCATTACCGCCAGTAATTCAACAATA } \\ \text { Bundle4_E } & \text { GGCCCTGAGAGAAGCAGGCGAAAATCATTGCGTAGAGGCGGT } \\ \text { Bundle10_E } & \text { CTTAAACAGCTTATATATTCGGTCGCTTGATGGGGAACAAGA } \\ \text { Bundle11_E } & \text { GCTCACAATTCCGTGAGCTAACTCACTGGAAGTAATGGTCAA } \\ \text { Bundle12_E } & \text { TTTGCGGATGGCCAACTAAAGTACGGGCTTGCAGCTACAGAG } \\ \text { Bundle1_F } & \text { GACAGGAGGTTGAAACAAATAAATCCGCCCCCTCCGCCACCC } \\ \text { Bundle2_F } & \text { CAGAATCAAGTTTCGGCATTTTCGGTTAAATATATCACCAGT } \\ \text { Bundle8_F } & \text { TCATATGGTTTACGATTGAGGGAGGGAAACGCAATACATACA } \\ \text { Bundle9_F } & \text { AATAGCAATAGCACCAGAAGGAAACCTAAAGCCACTGGTAAT }\end{array}$

Divalent cluster:

$\begin{array}{ll}\text { Name } & \text { Sequence (5'-3') } \\ \text { Bundle5_A } & \text { AGAGCCTAATTTGATTTTTGTTTAAATCCTGAAATAAAGAA } \\ \text { Bundle6_A } & \text { TGTAGCATTCCAACGTTAGTAAATGAAGTGCCGCGCCACCCT } \\ \text { Bundle9_A } & \text { GAAACATGAAAGCTCAGTACCAGGCGAAAAATGCTGAACAAA } \\ \text { Bundle10_A } & \text { TTTGCGGAACAATGGCAATTCATCAATCTGTATAATAATTTT } \\ \text { Bundle1_B } & \text { AAAGATTCATCAGGAATTACGAGGCATGCTCATCCTTATGCGAAAAAAAAAAAAAAA } \\ \text { Bundle6_B } & \text { CTTCATCAAGAGAAATCAACGTAACAGAGATTTGTCAATCATAAAAAAAAAAAAAA } \\ \text { Bundle7_B } & \text { CAAATGCTTTAAAAAATCAGGTCTTTAAGAGCAGCCAGAGGGAAAAAAAAAAAAAA } \\ \text { Bundle12_B } & \text { AAACGAAAGAGGGCGAAACAAAGTACTGACTATATTCGAGCTAAAAAAAAAAAAAA } \\ \text { Bundle2_C } & \text { AGCTTTCATCAACGGATTGACCGTAAAATCGTATAATATTTT } \\ \text { Bundle3_C } & \text { ACTGTTGGGAAGCAGCTGGCGAAAGGATAGGTCAAGATCGCA } \\ \text { Bundle7_C } & \text { GGTAGCTATTTTAGAGAATCGATGAAAACATTAAATGTGTAG } \\ \text { Bundle11_C } & \text { ATAAATCATACATAAATCGGTTGTACTGTGCTGGCATGCCTG } \\ \text { Bundle3_D } & \text { CAACGCTCAACAGCAGAGGCATTTTCAATCCAATGATAAATAAAAAAAAAAAAAAAA } \\ \text { Bundle4_D } & \text { ATCAAAATCATATATGTAAATGCTGAACAAACACTTGCTTCTAAAAAAAAAAAAAA } \\ \text { Bundle5_D } & \text { TGATTGCTTTGAGCAAAAGAAGATGAAATAGCAGAGGTTTTGAAAAAAAAAAAAAA } \\ \text { Bundle8_D } & \text { AACGGGTATTAAGGAATCATTACCGCCAGTAATTCAACAATAAAAAAAAAAAAAAA } \\ \text { Bundle4_E } & \text { GGCCCTGAGAGAAGCAGGCGAAAATCATTGCGTAGAGGCGGT } \\ \text { Bundle10_E } & \text { CTTAAACAGCTTATATATTCGGTCGCTTGATGGGGAACAAGA } \\ \text { Bundle11_E } & \text { GCTCACAATTCCGTGAGCTAACTCACTGGAAGTAATGGTCAA } \\ \text { Bundle12_E } & \text { TTTGCGGATGGCCAACTAAAGTACGGGCTTGCAGCTACAGAG } \\ \text { Bundle1_F } & \text { GACAGGAGGTTGAAACAAATAAATCCGCCCCTCCGCCACCC } \\ \text { Bundle2_F } & \text { CAGAATCAAGTTTCGGCATTTTCGGTTAAATATATCACCAGT } \\ \text { Bundle8_F } & \text { TCATATGGTTTACGATTGAGGGAGGGAACGCAATACATACA } \\ \text { Bundle9_F } & \text { AATAGCAATAGCACCAGAAGGAAACCTAAAGCCACTGGTAAT } \\ & \end{array}$


6.4 DNA for functionalization of QDs and AuNPs

$\begin{array}{ll}\text { Name } & \text { Sequence (5'-3') } \\ \text { Biotin-DNA for QDs } & \text { TAT GAA GTG ATG GAT GAT-biotin } \\ \text { Thiol-DNA for AuNPs } & \text { HS-TTTTTTTTTTTTTTTTTTTTTTTTTTTTTTT }\end{array}$

\section{References}

1. Tian, Y.; Wang, T.; Liu, W.; Xin, H. L.; Li, H.; Ke, Y.; Shih, W. M.; Gang, O. Prescribed Nanoparticle Cluster Architectures and Low-Dimensional Arrays Built Using Octahedral DNA Origami Frames. Nat. Nanotechnol. 2015, 10, 637-644.

2. Lakowicz, J. R. Principles of Fluorescence Spectroscopy, 3 ed.; Springer Science \& Business Media: New York, 2006; pp 443-448.

3. Maye, M. M.; Gang, O.; Cotlet, M. Photoluminescence Enhancement in Cdse/Zns-DNA Linked-Au Nanoparticle Heterodimers Probed by Single Molecule Spectroscopy. Chem. Commun. 2010, 46, 61116113.

4. Sun, D.; Tian, Y.; Zhang, Y.; Xu, Z.; Sfeir, M. Y.; Cotlet, M.; Gang, O. Light-Harvesting Nanoparticle Core-Shell Clusters with Controllable Optical Output. ACS Nano 2015, 9, 5657-5665.

5. Yun, C. S.; Javier, A.; Jennings, T.; Fisher, M.; Hira, S.; Peterson, S.; Hopkins, B.; Reich, N. O.; Strouse, G. F. Nanometal Surface Energy Transfer in Optical Rulers, Breaking the Fret Barrier. J. Am. Chem. Soc. 2005, 127, 3115-3119.

6. Jennings, T. L.; Singh, M. P.; Strouse, G. F. Fluorescent Lifetime Quenching near D $=1.5 \mathrm{Nm}$ Gold Nanoparticles: Probing Nset Validity. J. Am. Chem. Soc. 2006, 128, 5462-5467.

7. Pons, T.; Medintz, I. L.; Sapsford, K. E.; Higashiya, S.; Grimes, A. F.; English, D. S.; Mattoussi, H. On the Quenching of Semiconductor Quantum Dot Photoluminescence by Proximal Gold Nanoparticles. Nano Lett. 2007, 7, 3157-3164.

8. Singh, M. P.; Strouse, G. F. Involvement of the Lspr Spectral Overlap for Energy Transfer between a Dye and Au Nanoparticle. J. Am. Chem. Soc. 2010, 132, 9383-9391.

9. Breshike, C. J.; Riskowski, R. A.; Strouse, G. F. Leaving Förster Resonance Energy Transfer Behind: Nanometal Surface Energy Transfer Predicts the Size-Enhanced Energy Coupling between a Metal Nanoparticle and an Emitting Dipole. J. Phys. Chem. C 2013, 117, 23942-23949.

10. Chance, R. R.; Prock, A.; Silbey, R., Molecular Fluorescence and Energy Transfer near Interfaces. In Advances in Chemical Physics, Wiley-Blackwell: 2007; pp 1-65.

11. Haiss, W.; Thanh, N. T. K.; Aveyard, J.; Fernig, D. G. Determination of Size and Concentration of Gold Nanoparticles from Uv-Vis Spectra. Anal. Chem. 2007, 79, 4215-4221.

12. Johnson, P. B.; Christy, R. W. Optical Constants of the Noble Metals. Phys. Rev. B 1972, 6, 43704379.

13. Navarro, J. R. G.; Werts, M. H. V. Resonant Light Scattering Spectroscopy of Gold, Silver and GoldSilver Alloy Nanoparticles and Optical Detection in Microfluidic Channels. Analyst 2012, 138, 583-592. 